\title{
Coupling of Planar Waveguide Modes in All-Dielectric Multilayer Structures: Monitoring the Dependence of Local Electric Fields on the Coupling Strength
}

\author{
Kengo Motokura, ${ }^{1}$ Minoru Fujii $\odot,{ }^{1}$ Dmitry V. Nesterenko $\odot,{ }^{2,3}$ Zouheir Sekkat $\odot,{ }^{4,5,6}$ and \\ Shinji Hayashi $\odot^{1,4, *}$ \\ ${ }^{1}$ Department of Electrical and Electronic Engineering, Graduate School of Engineering, Kobe University, \\ Kobe 657-8501, Japan \\ ${ }^{2}$ Image Processing Systems Institute RAS - Branch of the FSRC "Crystallography and Photonics" RAS, Samara \\ 443001, Russia \\ ${ }^{3}$ Faculty of Information Technology, Samara National Research University, Samara 443086, Russia \\ ${ }^{4}$ Optics and Photonics Center, Moroccan Foundation for Science, Innovation and Research (MAScIR), University \\ Mohammed VI Polytechnic, Rabat 10100, Morocco \\ ${ }^{5}$ Faculty of Sciences, Mohamed V University in Rabat, Rabat 10010, Morocco \\ ${ }^{6}$ Graduate School of Engineering, Osaka University, Osaka 585-0871, Japan
}

(Received 30 June 2021; revised 25 August 2021; accepted 30 November 2021; published 28 December 2021)

Except for a few cases to which sophisticated techniques are applicable, it is difficult to observe experimentally local electric fields or near fields associated with excitation of electromagnetic modes supported by building blocks of nanostructures. Although elucidating the behavior of the local electric fields or near fields is very useful to develop potential applications of the nanostructures, in many cases, their behaviors have been only speculated from simulations based on the electromagnetic theory. Here, we develop a simple technique that allows us to study experimentally the average behavior of the local electric fields inside two planar waveguide layers interacting with each other in all-dielectric multilayer structures. The study is made possible by doping the two waveguide layers with two different fluorophores. Fluorescence intensities detected at two different wavelengths allow us to study separately the behaviors of the local electric fields inside each waveguide layer. Angle-scan fluorescence excitation spectra are measured for two different series of multilayer samples by exciting the samples in an attenuated total reflection geometry. The line shapes observed are in good agreement with theoretical ones calculated based on the electromagnetic theory, confirming that the experimental fluorescence excitation spectra reflect well the average behaviors of the local electric fields. Applying the developed technique, we investigate systematically the changes in the local electric fields inside the waveguide layers caused by the change in the strength of coupling between the two waveguide modes. In one series of the samples, a gradual transition in the behavior of the local electric fields, from a Fano-resonant behavior to a double-peak behavior, is demonstrated with increasing the coupling strength. In another series of the samples, the coupling strength is widely changed and the formation of the coupled modes in a strong coupling regime is clearly observed. The observed changes in the local electric fields can be reproduced very well by simple analytical calculations based on a coupled oscillator model. The model is helpful to gain physical insights into the coupling of the waveguide modes, which cannot be obtained by the electromagnetic calculations.

DOI: 10.1103/PhysRevApplied.16.064065

\section{INTRODUCTION}

In a variety of vibrational systems including mechanical, optical, and electrical systems, coupling between resonant

\footnotetext{
*s.hayashi@dragon.kobe-u.ac.jp

Published by the American Physical Society under the terms of the Creative Commons Attribution 4.0 International license. Further distribution of this work must maintain attribution to the author(s) and the published article's title, journal citation, and DOI.
}

modes plays a fundamental role in determining their vibrational properties, because the coupling modifies greatly the mode properties and leads to various alternative phenomena. In nanophotonics, the mode coupling has also been a subject of extensive experimental and theoretical studies. When the coupling is weak enough (weak coupling regime), it is well known that asymmetric line shapes in optical responses are generated from the coupling of a broad (bright) electromagnetic (EM) mode with a sharp (dark) EM mode [1-4]. The generation of the asymmetric line shape in far-field optical spectra can be explained 
in terms of constructive and destructive interference of pathways governed by the broad and sharp modes [5]; in analogy with the Fano resonance caused by the quantum interference between a continuum with a discrete state [6], the asymmetric line shape is called Fano line shape. As described in detail in recent review articles [1-3,7] and references therein, the Fano resonance has been studied extensively for a variety of nanostructures consisting of metallic and dielectric building blocks. Since the Fano line shapes contain steeper parts compared to the ordinary Lorentzian line shape, they can be used to improve the sensitivity of refractive index sensors [8,9] or the speed of optical switches [10].

Normally, Fano line shapes are reported for optical spectra measured as a function of photon energy (or wavelength) and represent the optical response in the space of $\omega$ (angular frequency of light). In contrast, planar multilayer structures integrated in angle-scan attenuated total reflection (ATR) configurations offer the possibility of studying the Fano resonance as a function of in-plane wave vector $k_{\|}$(for a fixed $\omega$ ), because the EM modes responsible for the optical responses of the structures are characterized by dispersion curves drawn in the $k_{\|}-\omega$ space. In fact, Fano line shapes in angle-scan ATR spectra of metal-insulatormetal $(M-I-M)$ structures are theoretically predicted by Grotewohl et al. [11,12] and experimentally demonstrated by Tomita et al. $[13,14]$. The observed Fano resonances originate from the coupling between a metal-clad dielectric waveguide mode (broad mode) and a surface plasmon polariton (SPP) mode at the outermost interface of the structures (sharp mode).

Over the past 5 years, we have been involved in the study of angle-scan ATR spectra of metal-dielectric and all-dielectric multilayer structures. For the metal-dielectric multilayer structures [15-18], we have shown that sharp Fano line shapes with high- $Q$ factors can easily be generated by letting a SPP mode (broad mode) at a metaldielectric interface couple with a sharp planar waveguide (PWG) mode supported by a dielectric waveguide layer; the $Q$ factor achieved was as high as approximately 1500 [18]. We have theoretically shown that the high- $Q$ Fano resonance realized in the multilayer structures can be applied to refractive-index sensing with a sensitivity much higher than that of the conventional surface plasmon resonance sensor [19,20]. For all-dielectric multilayer structures, we have shown that the Fano resonance can be generated by the coupling between two PWG modes (broad and sharp modes) supported by two dielectric waveguide layers [21]. By doping photochromic molecules into one of the dielectric waveguide layers in the Fano-resonant multilayer structures, we have succeeded in modulating the Fano line shape by illuminating the structures by external pump light [22-26].

When the coupling is so strong that the energy exchange rate between the uncoupled modes exceeds the decay rates of the modes (strong coupling regime), normal modes of the system turn to be two coupled modes formed by hybridizations of the uncoupled modes. Features commonly observed in the strong coupling regime are the increase in the splitting of the coupled modes with increasing the coupling strength and anticrossing behavior of the modes [27]. The light-matter interaction in the strong coupling regime has been studied for a long time $[28,29]$ and still constitutes a vast field of research [30-34]. In the field of light-matter interaction, the splitting is called Rabi splitting. In analogy with the splitting of atomic levels caused by strong pump light, the splitting is also referred to as Autler-Townes splitting [35] in the literature.

For a metal-insulator-metal multilayer structure consisting of a poly(methyl methacrylate) layer sandwiched by Ag layers, we have demonstrated the splitting and anticrossing behavior of the coupled modes formed by the coupling between two SPP modes [17,36]. Matsunaga et al. [14] also demonstrated similar anticrossing behaviors for another $M-I-M$ structure consisting of a $\mathrm{SiO}_{2}$ layer sandwiched by $\mathrm{Au}$ and $\mathrm{Ag}$ layers, and discussed a transition of ATR response from electromagnetically induced transparency (EIT), a special case of Fano resonance with zero detuning of uncoupled modes, to Autler-Townes splitting.

As pointed out in several papers [14,37-41], the Fano resonance and coupled-mode splitting sometimes show similar line shapes in far-field optical responses (e.g., scattering, extinction, and reflectance spectra) exhibiting a dip, which may lead to misunderstanding of underlying physics. The Fano dip is originated from the energy suppression of the broad mode due to the destructive interference with the sharp mode. In contrast, the dip associated with the coupled-mode splitting appears at a valley between two peaks. Discrimination of EIT from AutlerTownes splitting based on Akaike information criteria has been discussed in Refs. [14,38,40]. Although not mentioned in these papers, there is a possibility of distinguishing between the Fano resonance and the coupled-mode splitting from the behaviors of local electric fields or near fields associated with the building blocks that support the uncoupled modes. In the case of the Fano resonance, the intensity of local electric fields or near fields associated with the building block supporting a broad mode exhibits an asymmetric line shape with a dip, while that associated with the building block supporting a sharp mode exhibits a Lorentzian peak at the position of the Fano resonance (see, e.g., Fig. 3 in Ref. [39] and Fig. 5 in Ref. [21]). In contrast, in the case of the coupled-mode splitting, the intensities of local electric fields or near fields associated with both building blocks exhibit double-peak behaviors similar to those of the far-field response and do not exhibit a peak at the dip position (see Fig. 3 in Ref. [39]). Since the relation between the far-field response and the behavior of local electric fields or near fields is generally complex $[39,42,43]$, the study of far-field responses only is not 
sufficient to understand the effects of mode coupling on the line shape of the far-field spectra. Therefore, elucidating the behaviors of the local electric fields or near fields is extremely useful to clarify the mechanism of the line-shape formation in the far-field spectra. Unfortunately, except for rare cases to which sophisticated experimental techniques such as cathodoluminescence [44] and scanning near-field optical microscopy [45] are applicable, it is difficult to experimentally observe the behaviors of the local electric fields or near fields; they are usually speculated by simulations based on the EM theory. Acquiring detailed knowledge about the local electric fields and near fields is indispensable for developing optical devices based on their peculiar behaviors.

Very recently, we have attempted to generate Fano line shapes in fluorescence spectra of all-dielectric multilayer structures $[46,47]$ that support a broad PWG mode coupled with a sharp PWG mode. The strategy used was to dope fluorescent molecules into the waveguide layer supporting the broad PWG mode and monitor the fluorescence emitted by the molecules. The experimental fluorescence excitation [46] and emission [47] spectra measured in the angle-scan ATR geometry clearly showed the Fano line shapes, which agree well with theoretical ones calculated by the EM theory using distributions of local electric fields inside the waveguide layers. Although these studies suggested that the fluorescence measurements based on our strategy can be a versatile tool for investigating behaviors of local electric fields inside waveguides, such a possibility has not yet been fully explored. Furthermore, our main interest has been the generation of Fano line shape in the weak coupling regime. No systematic study was made so far on the effect of coupling strength in a wide range including the strong coupling regime.

In this work, extending the strategy used in our previous studies $[46,47]$, we develop a method that allows us to monitor simultaneously the behaviors of local electric fields inside both of the two waveguide layers coexisting in multilayer samples. The simultaneous monitoring is made possible by appropriately choosing two different kinds of fluorophores doped independently into the waveguide layers. The developed method is applied to study systematically the dependence of the local electric fields on the strength of coupling between the two PWG modes. We attempt to go beyond the Fano line shape entering into the strong coupling regime. Angle-scan fluorescence excitation spectra measured in an ATR configuration for two different series of samples are presented. From a comparison with electric field distributions calculated by the EM theory, we show that the experimental fluorescence spectra reflect well the behaviors of local electric fields. Furthermore, we show that changes in the fluorescence spectra caused by the change in the coupling strength can be well described by a simple model of two coupled oscillators. The coupled oscillator model, which is modified to take into account the spacer thickness dependence of the coupling strength, is shown to be very effective to analyze the behavior of the local electric fields in both the weak and strong coupling regimes. In a transition region from the weak to strong coupling regime, our results clearly demonstrate the deviation of the line shape from the Fano line shape. In the strong coupling regime, evidence is given for the generation of the symmetric and antisymmetric modes arising from the coupling of two waveguide modes.

\section{EXPERIMENTAL METHODS}

Figure 1(a) shows schematically the structure of multilayer samples prepared; this structure is essentially the same as that used in our previous studies $[21,46,47]$. The sample is composed of five organic polymer layers: a stack of three polyvinyl alcohol (PVA) layers and two polystyrene (PS) layers deposited on a SF11 glass substrate. Both of the PS layers are sandwiched by PVA layers and they work as cores of planar waveguides, because the refractive index of the PS core layers is larger than that of the PVA clad layers [48]. Hereafter, we denote these PS waveguide core layers as waveguide 1 (WG1) and waveguide 2 (WG2) layers, as indicated in Fig. 1(a). The WG1 layer is separated from the SF11 substrate by the first PVA spacer layer, referred to as the S1 layer, and the WG1 and WG2 layers are separated from each other by the second PVA spacer layer, referred to as the S2 layer. Both the WG1 and WG2 layers can support planar waveguide modes, referred to as PWG1 and PWG2 modes, respectively, which propagate inside the core layers along the in-plane direction $[x$ direction in Fig. 1(a)], accompanying evanescent waves inside the PVA clad layers. When the S2 spacer layer is thin enough, the PWG1 and PWG2 modes can couple each other through the overlap of the evanescent waves inside the S2 layer; the thinner the S2 layer, the stronger the coupling. In our previous studies, we have demonstrated that by doping the WG1 layer with fluorescent molecules and adjusting the structural parameters, Fano line shapes can be generated in the fluorescence excitation spectra [46] as well as in the fluorescence emission spectra [47], measured in an ATR configuration.

In our previous studies [46,47], only the WG1 layer was doped with fluorophores. However, in the present work, to study the local electric fields in both the WG1 and WG2 layers, the respective waveguide layers are doped with two different types of fluorophores. The fluorophores used are 4-(dicyanomethylene)-2-methyl-6-(4dimethylaminostyryl)-4H-pyran (DCM) for the WG1 layer and Coumarin 314 (C314) for the WG2 layer. Typical absorption and fluorescence spectra of a DCM-doped PS (DCM-PS) film and a C314-doped PS (C314-PS) film are shown in Fig. 1(b). The DCM-PS and C314-PS films show broad absorption bands peaking at 470 and $440 \mathrm{~nm}$, respectively; these bands overlap in the wavelength range 

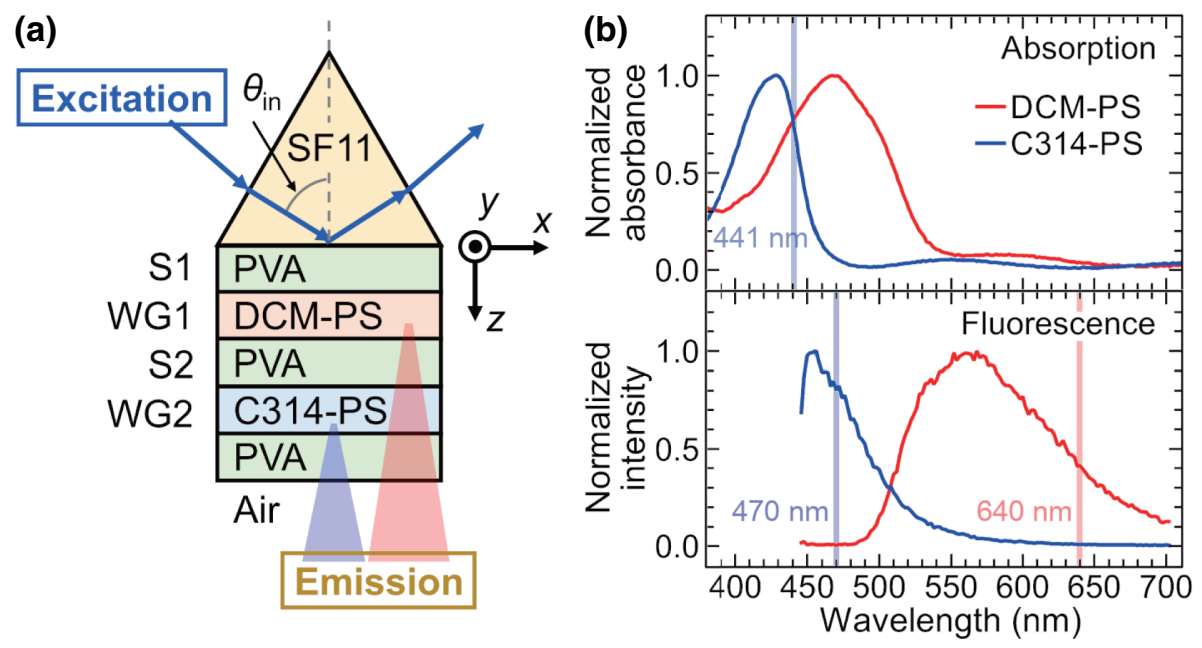

(c)

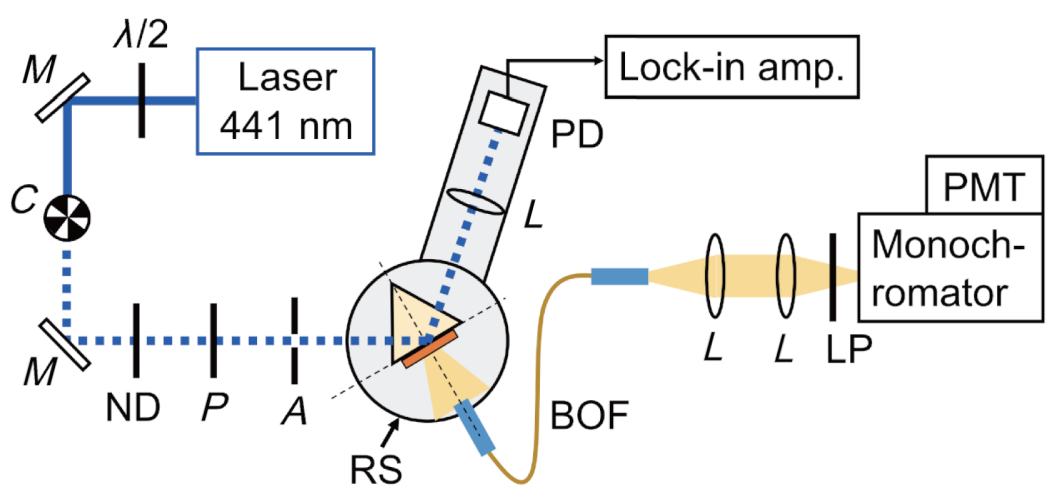

FIG. 1. (a) Structure of a five-layered sample consisting of PVA (S1), DCMPS (WG1), PVA (S2), C314-PS (WG2), and PVA layers. (b) Absorption and fluorescence spectra of C314-PS and DCM-PS layers. The spectra are normalized by their maximum values. (c) Optical system used to measure anglescan ATR and fluorescence excitation spectra. $\lambda / 2$, half-wave plate; $M$, mirror; $C$, chopper; ND, neutral density filter; $P$, polarizer; $A$, aperture; RS, rotation stage; $L$, lens; PD, photodiode; BOF, bundle optical fiber; LP, long pass filter; PMT, photomultiplier tube. Excitation wavelength is $\lambda=441 \mathrm{~nm}$. Observation wavelengths to monitor fluorescence from DCM-PS and C314-PS layers are set to be $\lambda=640$ and $470 \mathrm{~nm}$, respectively. The excitation and observation wavelengths are represented by vertical lines in (b). shorter than approximately $470 \mathrm{~nm}$. The DCM-PS and C314-PS films show broad fluorescence bands peaking around 560 and $460 \mathrm{~nm}$, respectively; in the green region $(480 \mathrm{~nm}<\lambda<570 \mathrm{~nm})$, these bands overlap each other, but outside the overlap region, for $\lambda<480 \mathrm{~nm}$ only the C314-PS film exhibits strong enough signals, while for $\lambda>600 \mathrm{~nm}$, it is the DCM-PS film that shows the strong enough signals. The overlap of the absorption bands allows us to excite simultaneously the fluorescence of the WG1 and WG2 layers by selecting the excitation wavelength falling in the overlap region. On the other hand, the existence of the nonoverlapping regions of the fluorescence bands allows us to detect separately the fluorescence signals from the C314-PS and DCM-PS layers. Namely, by choosing the detection wavelength in the region of $\lambda<480 \mathrm{~nm}$, we can detect the signal from the WG2 layer, while by choosing the wavelength in the region of $\lambda>600 \mathrm{~nm}$, we can detect the signal from the WG1 layer.

All the samples are fabricated by a spin-coating method on cleaned SF11 substrates. The details of the fabrication process are described in the Supplemental Material [49]. As shown in Fig. 1(a), the multilayer sample is pasted onto a $60^{\circ}$ prism made of SF11 with the aid of index matching fluid to construct an ATR configuration. Using the optical setup schematically shown in Fig. 1(c), we measure angle-scan ATR spectra and fluorescence spectra excited in the ATR configuration. A s-polarized excitation beam approximately $1 \mathrm{~mm}$ in diameter from a semiconductor laser (Civil Laser) with a wavelength of $441 \mathrm{~nm}$ is incident on the sample through the prism; note that this excitation wavelength falls in the overlap region of the C314-PS and DCM-PS absorption bands. The angle of incidence $\theta_{\text {in }}$ is varied by rotating a stage on which the prism with the sample is mounted. The power of the excitation light is set to $20 \mu \mathrm{W}$; the estimated power density is approximately $2.5 \mathrm{~mW} / \mathrm{cm}^{2}$. To obtain the ATR spectra, the intensity of the reflected light exiting from the prism is measured by a photodiode fixed on a rotation arm. The fluorescence emitted into the air side of the sample is collected from the normal direction ( $z$ direction) by a bundle fiber fixed on the rotation stage approximately $15 \mathrm{~mm}$ away from the sample surface and guided to a monochromator equipped with a photomultiplier tube (PMT) (Hamamatsu Photonics, H7421-40); the fluorescence intensity is measured by a photon-counting method. The observation wavelength is set either at $470 \mathrm{~nm}$ or at $640 \mathrm{~nm}$ to detect separately the fluorescence signals from the WG2 and WG1 layers, respectively. Normally, fluorescence excitation spectra are obtained by measuring the fluorescence intensity at a fixed wavelength, while varying the excitation wavelength. In the present fluorescence measurements, since the detection wavelength is fixed and the excitation angle $\theta_{\text {in }}$ 
is varied, it is appropriate to call the resulting spectra as the angle-scan fluorescence excitation spectra. It is worthwhile to notice that under the measurement conditions described above, the fluorescence excitation is mediated by the excitation of the waveguide modes (resonant excitation), while the emission is not mediated by the excitation of the waveguide modes (nonresonant emission).

We also prepare reference samples consisting of a DCMPS or a C314-PS single layer deposited on a SF11 substrate with the same thickness and dye concentration as those in the multilayer samples. Using the same optical setup and exciting the layer from the substrate side (without prism) at an angle of $45^{\circ}$, we measure the fluorescent intensity, which is used as the reference intensity. In the present work, we define the fluorescence enhancement factor (FLEF) as the fluorescence intensity of the multilayer sample divided by the reference intensity. We convert all the measured fluorescence excitation spectra to the spectra of FLEF. For all the samples, the experimental ATR spectra are fitted to theoretical ones using a Winspall software package [50], which allows us to calculate the ATR spectra by varying the structural parameters based on the EM theory of multilayer systems. As in our previous works $[16,18,21,25,26]$, the structural parameters necessary for EM analyses of the experimental results are determined from the fitting procedures.

To study systematically the behaviors of local electric fields by varying the strength of mode coupling, which can be controlled by the thickness of the S2 layer, we prepare two different series of multilayer samples. The sample preparation parameters and structural parameters are summarized in the Supplemental Material [49]. In a series of the samples, we manage to generate Fano-resonant behaviors of electric fields inside the WG1 layer. To realize a broad PWG1 mode and a sharp PWG2 mode the concentration of the DCM molecules inside the WG1 layer is set to be larger than that of $\mathrm{C} 314$ molecules inside the WG2 layer by a factor of approximately 37 , which results in an extinction coefficient of the WG1 layer approximately 14 times larger than that of the WG2 layer (see Tables S1 and S2 within the Supplemental Material [49]). The samples having different S2 thicknesses are prepared, while trying to keep the other structural parameters unchanged. Since the samples belonging to this series support a broad (B) PWG1 mode and a sharp (S) PWG2 mode, this series of samples is called BS series. In another series of samples, called SS series, we try to clarify the behavior of local electric fields by letting both the PWG1 and PWG2 modes be sharp and changing the coupling strength in a wide range. To make the PWG1 mode sharper than that of the BS sample series, the concentration of the DCM molecules inside the WG1 layer is set to be approximately 6.5 times smaller than that in the BS sample series, while keeping the concentration of the $\mathrm{C} 314$ molecules inside the WG2 layer the same as in the BS sample series. The thickness of the S2 layer is systematically varied in a range wider than that for the BS sample. The sample preparation parameters and structural parameters for the SS samples are found in Tables S1 and S3 within the Supplemental Material [49], respectively.

\section{EXPERIMENTAL RESULTS AND ELECTROMAGNETIC ANALYSES}

\section{A. Results for BS samples}

Figures 2(a)-2(c) show experimental FLEF spectra of three samples in the BS series. The S2 layer thicknesses of the samples are estimated from the fitting of measured ATR spectra to theoretical ATR spectra; experimental ATR spectra and fitting parameters (structural parameters of the samples) are given within the Supplemental Material (Fig. S1 and Table S2) [49]. The estimated S2 layer thicknesses for the samples are 380, 186, and $97 \mathrm{~nm}$, respectively. These samples are referred to as BS380, BS186, and BS97, respectively. As can be seen in Fig. 2(a), the FLEF spectrum of the sample BS380 observed at $640 \mathrm{~nm}$ exhibits a broad peak (approximately $60.5^{\circ}$ ) accompanied by a sharp dip located around $60.8^{\circ}$; the line shape seen around the dip is highly asymmetric, which is typical of the Fano resonance. On the other hand, the FLEF spectrum observed at $470 \mathrm{~nm}$ exhibits a sharp Lorentzian single peak around $60.8^{\circ}$, i.e., the dip position of the $640-\mathrm{nm}$ spectrum. In addition, the fluorescence enhancement factors achieved at the peaks are approximately 10 for the $640-\mathrm{nm}$ spectrum and approximately 73 for the $470-\mathrm{nm}$ spectrum, respectively.

When the thickness of the S2 layer is decreased, the FLEF spectrum is greatly modified. Figure 2(b) for the BS186 sample shows that the 640-nm spectrum also exhibits a dip around $60.8^{\circ}$, but it is wider and deeper than that of BS380. The broadening of the dip is more pronounced for BS97 having a thinner S2 layer, as can be seen in Fig. 2(c). Furthermore, the dip seen for BS97 appears to be less asymmetric and tends to be a valley located between two peaks at approximately $60.2^{\circ}$ and approximately $61.0^{\circ}$. This suggests that the line shape starts to deviate from the Fano line shape at a S2 thickness around $97 \mathrm{~nm}$. Along with the modification of the $640-\mathrm{nm}$ spectrum, the 470-nm FLEF spectrum also changes considerably depending on the S2 thickness. Figures $2(\mathrm{~b})$ and 2(c) demonstrate that the Lorentzian peak, initially located at approximately $60.8^{\circ}$ for BS380, broadens and slightly shifts to a higher angle, as the S2 thickness decreases. In addition, a broad peak emerges at the low-angle side of the main peak for BS186, and grows and shifts to a lower angle for BS97. The modifications of the Fano and Lorentzian line shapes seen in Fig. 2 are thought to be caused mainly by the increase in the strength of coupling between the PWG1 and PWG2 modes. In the next section, we analyze 

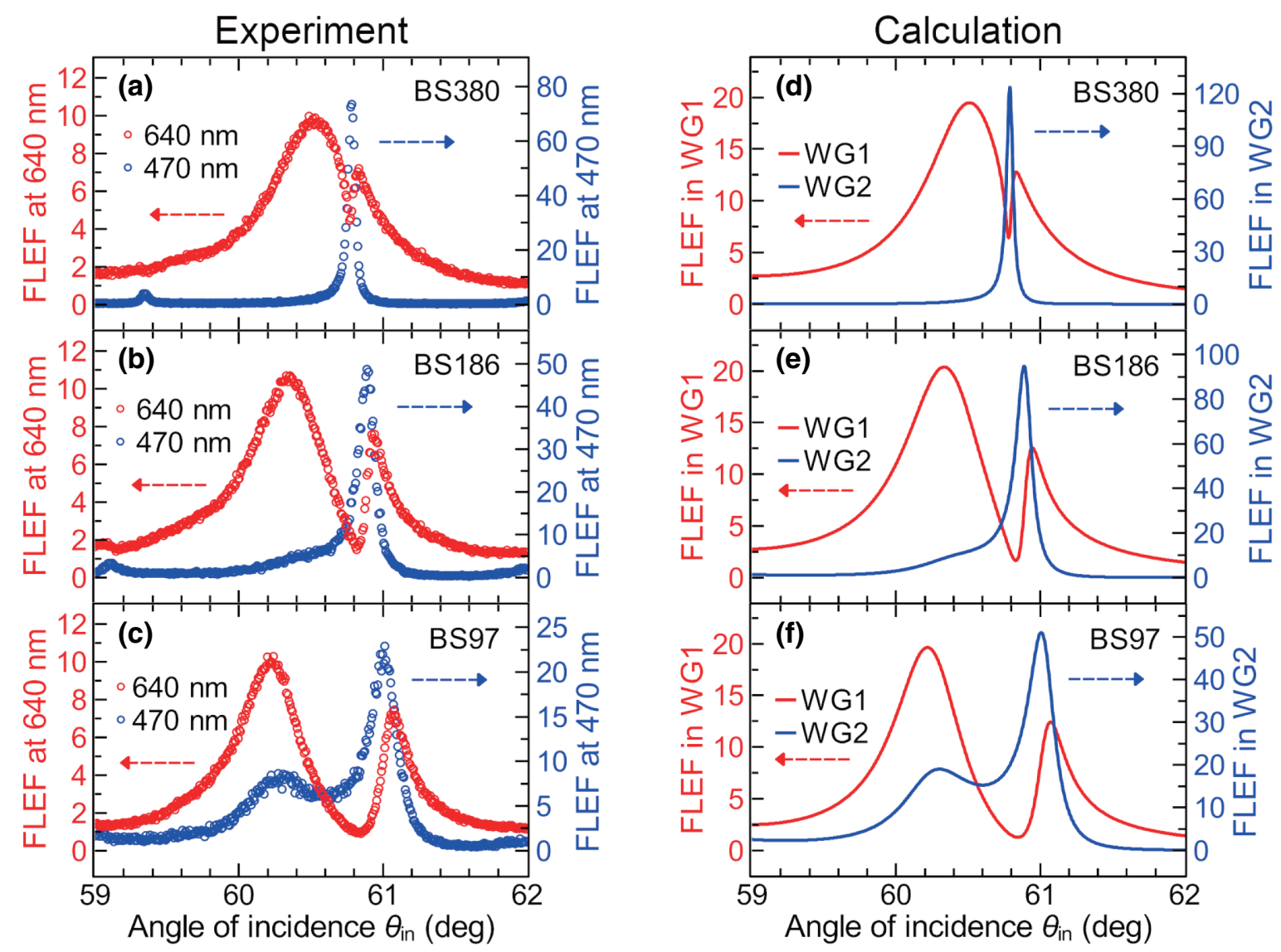

FIG. 2. Spectra of fluorescence enhancement factor (FLEF) for BS samples with S2 spacer thicknesses of 380, 186, and $97 \mathrm{~nm}$ (BS380, BS186, and BS97 samples). (a)-(c) Experimental FLEF spectra monitored at wavelengths of 470 and $640 \mathrm{~nm}$. (d)-(f) Theoretical FLEF spectra calculated for WG1 and WG2 layers.

in more detail the modification of the Fano line shape based on the coupled oscillator model.

We find that the 470-nm FLEF spectrum in Fig. 2(a) exhibits a tiny peak around $59.3^{\circ}$ and the spectrum in Fig. 2(b) also exhibits a tiny peak around $59.1^{\circ}$. From the fact that these tiny peaks are located close to the mirror positions of the main peaks with respect to $\theta_{\text {in }}=60^{\circ}$, we can attribute these peaks to the fluorescence signals excited by the incident light, first reflected at the prismsample interface, and secondly reflected at the prism-air interface and sent back to the prism-sample interface with an incidence angle of $120^{\circ}-\theta_{\text {in }}$. Since these tiny peaks are artifacts caused by the $60^{\circ}$ prism, they are excluded from the following discussions.

To confirm that the observed changes in the experimental FLEF spectra presented above really reflect the changes in the local electric fields inside the WG1 and WG2 layers, we compare them with theoretical ones. Our EM calculations are based on a point dipole model that has been used very frequently to discuss the fluorescence spectra of emitters embedded in a variety of multilayer systems $[46,51,52]$. In general, the intensity of fluorescence is determined by both the excitation and emission processes. When a fluorescence molecule is embedded in one of the layers in a multilayer structure and has an absorption transition dipole moment $\mathbf{p}_{a}$, the excitation rate is proportional to $\left|\mathbf{p}_{a} \cdot \mathbf{E}_{\text {loc }}\right|^{2}$, where $\mathbf{E}_{\text {loc }}$ is the local electric field at the position of the molecule $\mathbf{r}$ generated by the excitation light. For an ensemble of randomly oriented molecules, the excitation rate becomes proportional to $\left|\mathbf{E}_{\text {loc }}\right|^{2}$ after taking the rotational average. From these considerations, taking the $z$ axis normal to the interfaces as shown in Fig. 1(a), the excitation rate of fluorescence molecules lying on a plane at $z=z_{0}$ in the present multilayer structure is given by, apart from a proportionality constant, the field enhancement factor (FEF) $F_{\text {ex }}\left(\theta_{\text {in }}, \lambda_{\text {ex }}\right.$, $z_{0}$ ) defined as $\left|E\left(z_{0}\right)\right|^{2} /\left|E_{0}\right|^{2}$, where $E\left(z_{0}\right)$ is the local electric field at $z=z_{0}$ and $E_{0}$ is the electric field amplitude of excitation light. For our multilayer structures, since the thicknesses and refractive indices of the layers at the wavelength of excitation light $(441 \mathrm{~nm})$ are known from the theoretical fit of experimental ATR spectra, $F_{\text {ex }}\left(\theta_{\text {in }}, \lambda_{\text {ex }}\right.$, $z_{0}$ ) can be calculated assuming a plane wave for the excitation light and using a $2 \times 2$ transfer-matrix method [53]. Writing the intensity of the excitation light as $I_{0}$, which is proportional to $\left|E_{0}\right|^{2}$, the excitation rate can finally be 
written as $A F_{\text {ex }}\left(\theta_{\text {in }}, \lambda_{\text {ex }}, z_{0}\right) I_{0}$, where $A$ is a proportionality constant. As in the experiments, a s-polarized incident wave is assumed in the calculation of FEF.

Within the point dipole model, the light-emission process can be described by considering radiation fields emitted by a dipole oscillating at the emission frequency. As described in detail in Refs. [51,52], when the radiating dipole is placed in one of the layers of the multilayer structure, the light wave emitted repeats the reflection and transmission at the interfaces inside the structure, and the light observed in a free space outside the multilayer structure is a consequence of interference of the multiply reflected and transmitted light waves exiting from the structure. An explicit expression of the power density of the observed light is found in Refs. [51,52] and can be calculated using the reflection and transmission coefficients at the interfaces. In the present work, in the same manner as in our previous paper [46], we use an emission function $P_{\mathrm{em}}\left(\theta_{\mathrm{obs}}, \lambda_{\mathrm{em}}, z_{0}\right)$ defined as the ratio of the light power emitted by an emitter located at $z=z_{0}$ inside a layer into a unit solid angle around the observation point to the total power emitted by an emitter placed in an infinite medium having the refractive index of the layer material; $\lambda_{\text {em }}$ and $\theta_{\text {obs }}$ are the emission wavelength and observation angle, respectively. $P_{\mathrm{em}}\left(\theta_{\mathrm{obs}}, \lambda_{\mathrm{em}}, z_{0}\right)$ can be calculated when the thicknesses and refractive indices at the emission wavelength of the layers are known.

The emitted light is normally collected through an aperture with a finite size. When a small aperture is placed sufficiently far from the emission spot, which is the case in our experiments, the variation of $P_{\mathrm{em}}\left(\theta_{\mathrm{obs}}, \lambda_{\mathrm{em}}, z_{0}\right)$ in the aperture area caused by the deviation of $\theta_{\text {obs }}$ from the central value is negligibly small. In this case, the integration of $P_{\mathrm{em}}\left(\theta_{\mathrm{obs}}, \lambda_{\mathrm{em}}, z_{0}\right)$ over the finite solid angle of collection reduces to $B P_{\mathrm{em}}\left(\theta_{\mathrm{obs}}, \lambda_{\mathrm{em}}, z_{0}\right)$, where $B$ is a proportionality constant determined by the aperture size and the distance from the emission spot. Assuming that randomly oriented fluorescent molecules are distributed over the layer with a number density $D(z)$ and taking into account all the factors explained above, we can express the intensity of the fluorescence originating from the layer in an integral form,

$$
\begin{aligned}
& I_{\text {layer }}\left(\theta_{\text {in }}, \theta_{\text {obs }}, \lambda_{\text {ex }}, \lambda_{\mathrm{em}}\right)=C\left(\cos \theta_{\text {in }}\right)^{-1} I_{0} \\
& \quad \times \int_{\text {layer }} F_{\text {ex }}\left(\theta_{\text {in }}, \lambda_{\text {ex }}, z\right) P_{\mathrm{em}}\left(\theta_{\text {obs }}, \lambda_{\text {em }}, z\right) D(z) d z,
\end{aligned}
$$

where $C$ is a proportinality constant given by a product of the proportinality constants in the excitation and emission processes and $\left(\cos \theta_{\text {in }}\right)^{-1}$ accounts for the dependence of the excitation power per unit area on $\theta_{\text {in }}$. The integration is taken over the thickness of the layer containing the fluorescent molecules. In our experiments, $\lambda_{\mathrm{ex}}=441 \mathrm{~nm}$, $\lambda_{\mathrm{em}}=470$ and $640 \mathrm{~nm}$ for the WG2 and WG1 layers, respectively, and $\theta_{\mathrm{obs}}=0^{\circ}$. Equation (1) together with these values allows us to calculate the fluorescence excitation spectra $I_{\text {layer }}\left(\theta_{\text {in }}\right)$ as a function of $\theta_{\text {in }}$. The resonant behaviors of the local electric fields associated with the excitation of the waveguide modes are expected to appear in $I_{\text {layer }}\left(\theta_{\text {in }}\right)$ through $F_{\text {ex }}\left(\theta_{\text {in }}, \lambda_{\text {ex }}, z_{0}\right)$.

In our numerical calculations, for simplicity, $D(z)$ is assumed to be a constant, written as $D_{0}$. As mentioned above, $F_{\text {ex }}\left(\theta_{\text {in }}, \lambda_{\text {ex }}, z_{0}\right)$ is calculated by a $2 \times 2$ transfermatrix method using known values of the structural parameters. Unfortunately, values of the refractive indices of the layers at the emission wavelengths are not known experimentally. Therefore, we assume values of the refractive indices obtained by extrapolating the literature values [48]. To compare with experimental FLEF spectra, we calculate the fluorescence excitation spectra of the multilayer samples as well as the fluorescence intensity of the reference samples using Eq. (1) and take the ratios to obtain theoretical FLEF spectra. Since the constant $C, I_{0}$, and $D_{0}$ are common for both the multilayer and reference samples, the proportionality factor $C I_{0} D_{0}$ appearing in Eq. (1) is eliminated when the ratios of the intensities are taken to obtain the FLEF spectra.

Figures 2(d)-2(f) show theoretical FLEF spectra calculated by Eq. (1) for the samples BS380, BS186, and BS97, respectively. Values of the refractive indices to calculate $P_{\mathrm{em}}\left(\theta_{\mathrm{obs}}, \lambda_{\mathrm{em}}, z_{0}\right)$ are listed in Table S4 within the Supplemental Material [49]. We see that the line shapes of experimental FLEF spectra monitored at 640 and $470 \mathrm{~nm}$ are very well reproduced by the theoretical FLEF spectra obtained from FEF in the WG1 and WG2 layers, respectively. The magnitudes of FLEF in the experimental spectra are smaller than those in the theoretical ones by a factor of approximately 2 . Although the exact origin of this discrepancy is not known at present, we can point out several possible origins. First, the sample imperfections such as interface roughness and bulk inhomogeneity in the layers cause undesirable scattering loss in both the excitation and emission processes in the multilayer samples and lead to the decrease in the experimental FLEF. Secondly, the experiments are performed using an excitation laser beam with a finite cross section, while a plane wave with an infinite extent is assumed for the excitation light in the electromagnetic calculations. Therefore, effects arising from the finite cross section are not taken into account in the theoretical calculations. Thirdly, the values of optical constants at the emission wavelengths used for calculating the emission functions are extrapolated from the literature values and consequently, they may deviate to some extent from real values of the samples, leading to errors in the calculated fluorescence intensities. To clarify the exact origin of the discrepancy, further experimental and theoretical studies are required.

During the course of our analysis, we find that, as far as the relative intensities and spectral shapes are concerned, the position dependence of $P_{\mathrm{em}}\left(\theta_{\mathrm{obs}}, \lambda_{\mathrm{em}}, z_{0}\right)$ does not influence appreciably the results of calculations 
made by Eq. (1). We perform approximate calculations of $I_{\text {layer }}\left(\theta_{\text {in }}, \theta_{\text {obs }}, \lambda_{\text {ex }}, \lambda_{\text {em }}\right)$ by simply assuming $P_{\text {em }}\left(\theta_{\text {obs }}\right.$, $\left.\lambda_{\text {em }}, z_{0}\right)=1$ and compare the results with those obtained using the $P_{\mathrm{em}}\left(\theta_{\mathrm{obs}}, \lambda_{\mathrm{em}}, z_{0}\right)$ functions, after normalizing the intensities to their maximum values. As summarized within the Supplemental Material [49], a close comparison between these results reveals that both results are almost identical; the differences between the normalized spectra are much smaller than noise levels in the fluorescence intensity measurements. Based on this finding, we can replace Eq. (1) by a simple approximate form:

$$
\begin{aligned}
& I_{\text {layer }}\left(\theta_{\text {in }}, \theta_{\text {obs }}, \lambda_{\text {ex }}, \lambda_{\text {em }}\right)=C^{\prime}\left(\cos \theta_{\text {in }}\right)^{-1} I_{0} \\
& \quad \times \int_{\text {layer }} F_{\text {ex }}\left(\theta_{\text {in }}, \lambda_{\text {ex }}, z\right) d z
\end{aligned}
$$

where $C^{\prime}$ is a new proportionality constant. This equation implies that the fluorescence intensity is proportional to the integral of the field enhancement factor over the layer containing the fluorophores. As explained in detail in Sec. III of the Supplemental Material [49], Eq. (2) is valid when the fluorescence emission process is nonresonant with the waveguide modes. Since the fluorescence intensity obtained by Eq. (1) is well reproduced by Eq. (2) apart from the proportionality constant, we can finally conclude that the experimental FLEF spectra that agree with the theoretical ones, also reflect the integral or average behavior of the local electric fields.

Figures 3(a)-3(c) present the maps of FEF used in the calculations of the FLEF spectra. These maps are helpful to visualize the changes in the local electric fields inside the WG1 and WG2 layers that lead to the observed changes in the FLEF spectra. Figure 3(a), for the sample BS380, demonstrates that the local electric fields inside the WG1 layer exhibit a broad distribution extending from 59.5 to $61.5^{\circ}$, which correlates very well with the broad WG1 FLEF bands (640 nm) in Figs. 2(a) and 2(d). This broad distribution corresponds to the excitation of a $\mathrm{TE}_{0}$ waveguide mode inside the WG1 layer (PWG1 mode). The resonance of the PWG1 mode in the BS sample series is broad, because the extinction coefficient in the WG1 layer is large, resulting in a large intrinsic loss. In contrast, a very narrow field distribution is generated in the WG2 layer at approximately $60.8^{\circ}$, which correlates well with the very sharp Lorentzian peaks in the WG2 FLEF spectra $(470 \mathrm{~nm})$ seen in Figs. 2(a) and 2(d). The narrow field distribution in the WG2 layer corresponds to the excitation of a $\mathrm{TE}_{0}$ waveguide mode inside the WG2 layer (PWG2 mode) mediated by the near-field coupling to the PWG1 mode. Due to the coupling, a part of EM energy associated with the PWG1 mode is transferred to the PWG2 mode and consequently, electric fields inside the WG1 layer are greatly suppressed around the excitation angle of the PWG2 mode; the suppression of the fields in the WG1 layer thus leads

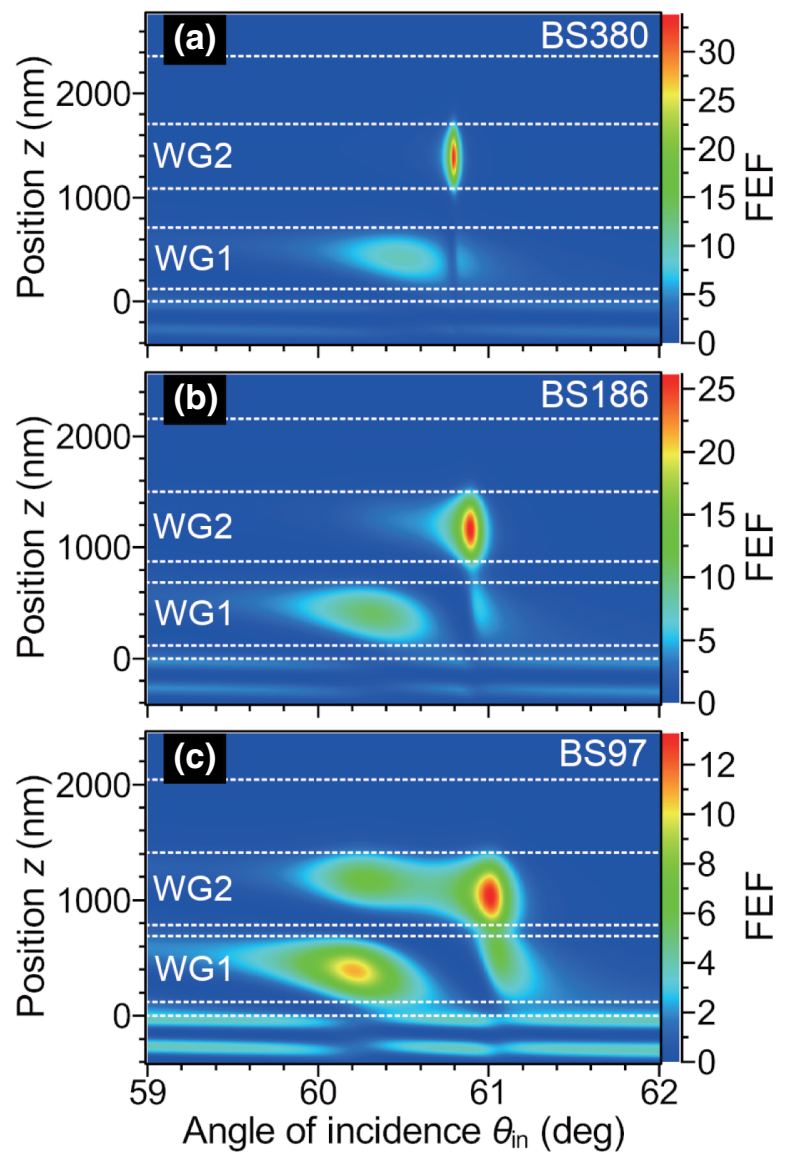

FIG. 3. Maps of theoretical electric field distributions (field enhancement factor, $\mathrm{FEF}=|E|^{2} /\left|E_{0}\right|^{2}$ ) calculated for BS samples, BS380 (a), BS186 (b), and BS97 (c).

to the Fano dip in the WG1 FLEF spectra. It should be noted that the mechanism of the Fano dip generation in the WG1 FLEF spectra is analogous to a widely accepted mechanism of Fano line shape generation in a variety of nanostructures, i.e., near-field coupling between a bright mode and a dark mode [1-4]; in our samples, the broad PWG1 mode plays the role of the bright mode, while the sharp PWG2 mode plays the role of the dark mode.

For the samples BS186 and BS97 with thinner S2 layers, i.e., having larger coupling strengths, the field distributions are greatly modified as can be seen in Figs. 3(b) and 3(c). In the WG1 layer, the broad distribution is clearly separated into two parts, and the separation becomes more pronounced as the S2 thickness decreases. On the other hand, in the WG2 layer, a sidelobe emerges at the lowangle side of the sharp distribution and grows as the S2 thickness decreases. These modifications in the field distributions caused by the increase in the coupling strength are origins of the line-shape changes in the FLEF spectra.

\section{B. Results for SS samples}

Figures 4(a)-4(d) show experimental FLEF spectra obtained for four different samples in the SS sample series, 


\section{Experiment}

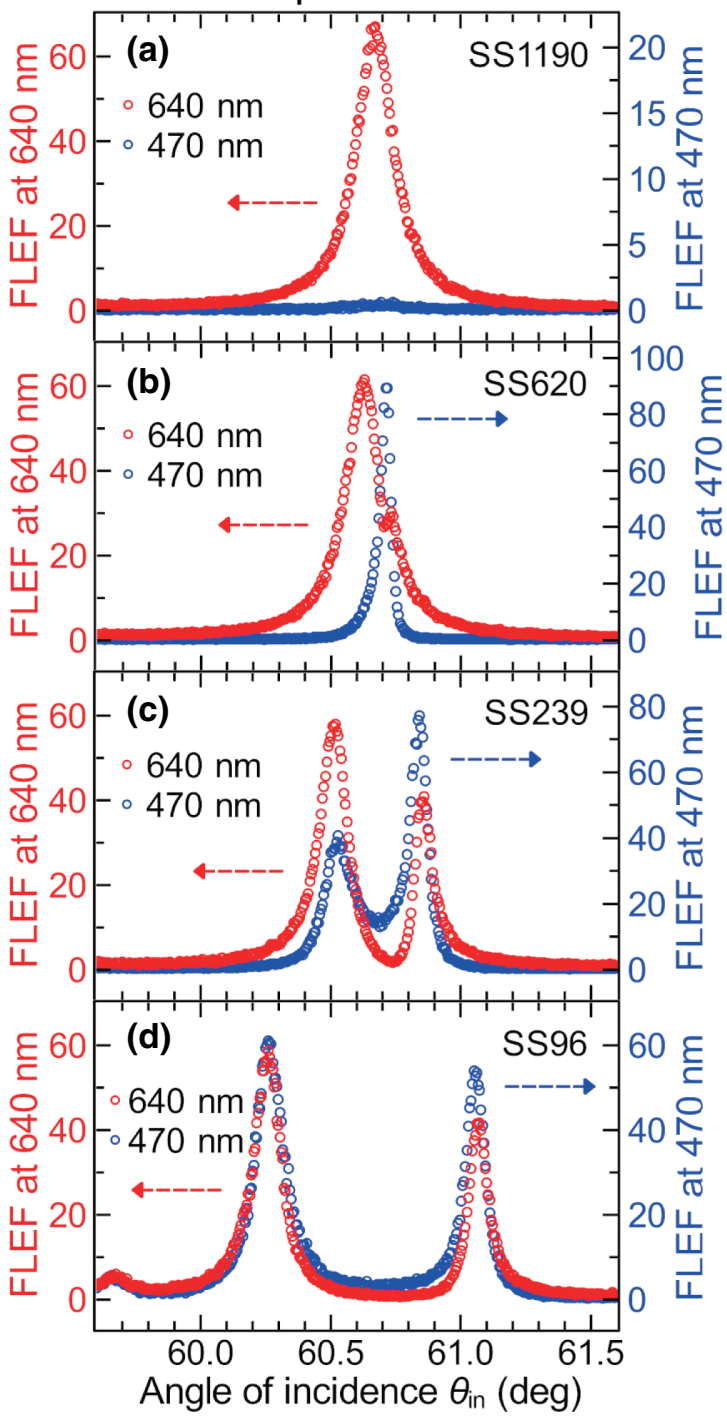

Calculation

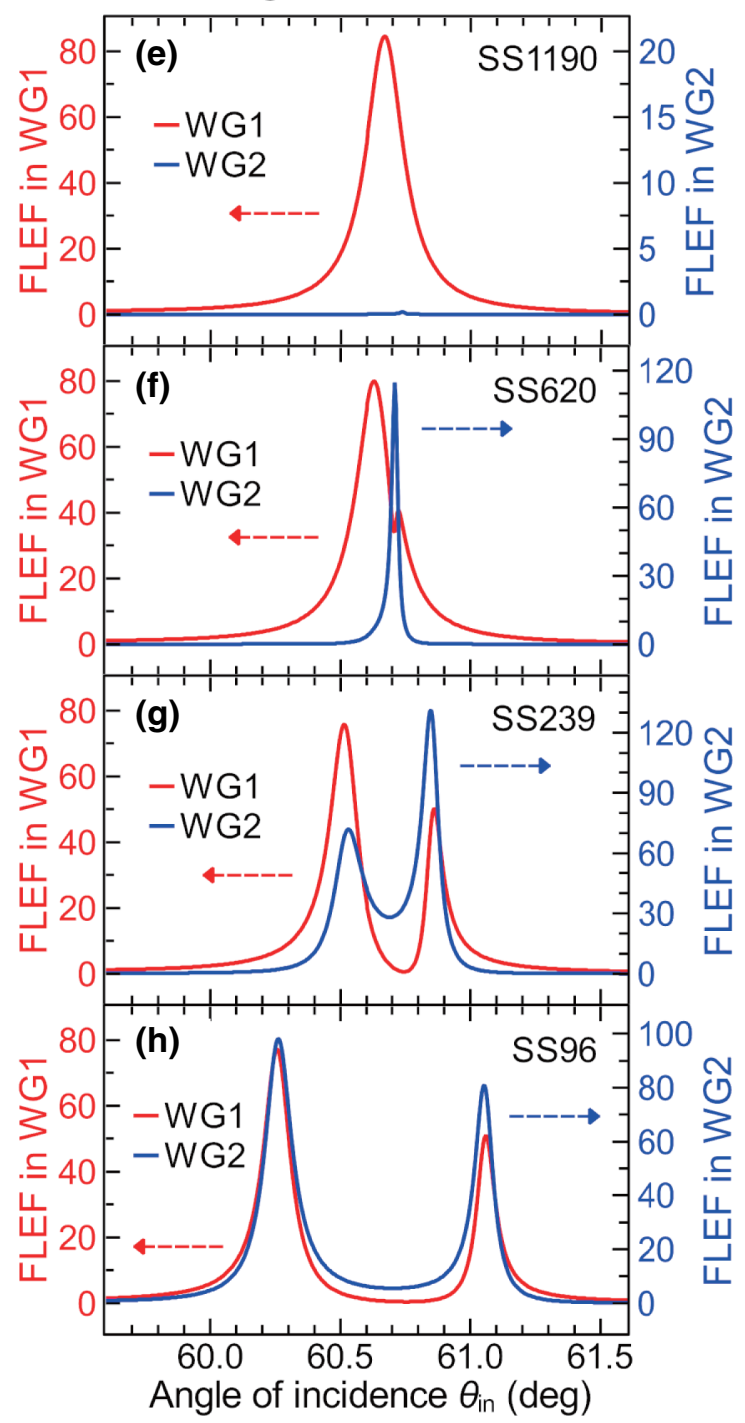

FIG. 4. FLEF spectra for SS samples with S2 spacer thickness of 1190, 620, 239, and $96 \mathrm{~nm}$ (SS1190, SS620, SS239, and SS96 samples). (a)-(d) Experimental FLEF spectra monitored at wavelengths of 470 and $640 \mathrm{~nm}$. (e)-(h) Theoretical FLEF spectra calculated for WG1 and WG2 layers.

in which the concentrations of fluorophores in both the WG1 and WG2 layers are low and the PWG1 and PWG2 resonances are expected to be sharp. Note that the span of incidence angle for Figs. 4(a) 4 (d) $\left(2.0^{\circ}\right)$ is $2 / 3$ of that for Figs. 2(a)-2(c) $\left(3.0^{\circ}\right)$. The S2 layer thicknesses of the SS samples estimated from the EM fitting of the ATR spectra are 1190, 620, 239, and $96 \mathrm{~nm}$, respectively, and these samples are referred to as SS1190, SS620, SS239, and SS96, respectively. The structural parameters of these samples are listed in Table S3 of the Supplemental Material [49]. Theoretical WG1 and WG2 FLEF spectra of the SS samples calculated in the same manner as those of the BS samples are presented in Figs. 4(e)-4(h), and maps of the field distributions used in these calculations are shown in Figs. 5(a)-5(d).
Figure 4(a) shows that the 640-nm FLEF spectrum of the sample SS1190 exhibits a single Lorentzian peak at approximately $60.7^{\circ}$, while the $470-\mathrm{nm}$ spectrum shows no significant signal. In the 470-nm FLEF spectrum for the sample SS620 presented in Fig. 4(b), a Lorentzian peak emerges at approximately $60.7^{\circ}$, and around the same angle, the 640-nm spectrum shows a very shallow dip. For both the SS239 and SS96 samples, the FLEF spectra shown in Figs. 4(c) and 4(d) exhibit double peaks for both emission wavelengths and the splitting of the peaks increases as the $\mathrm{S} 2$ thickness decreases. Comparing Figs. 4(a)-4(d) with Figs. 4(e)-4(h) we see that the experimental line shapes and their changes depending on the S2 thickness are very well reproduced by the theoretical spectra, although the amplitudes of FLEF in the theoretical 


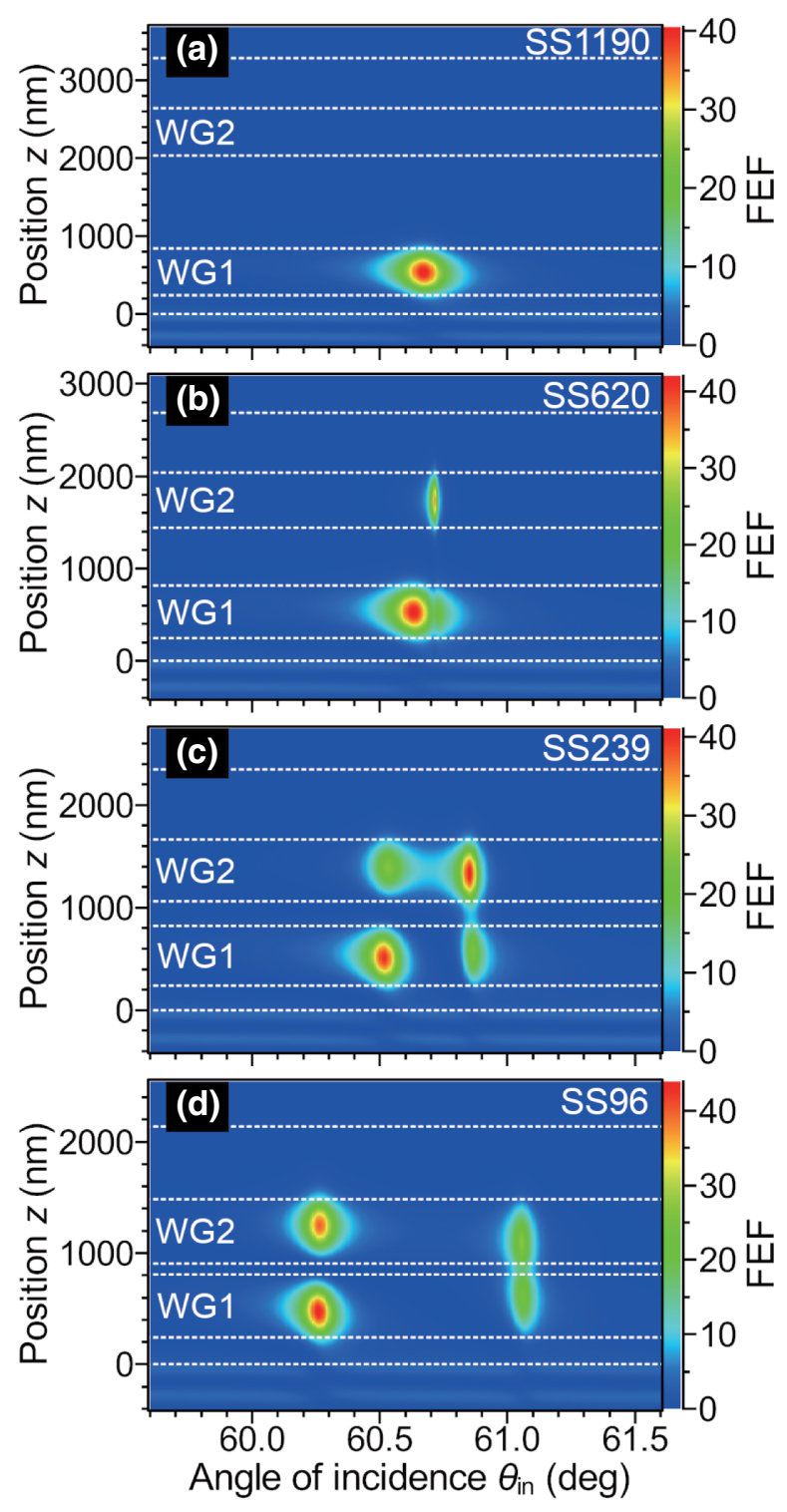

FIG. 5. Maps of theoretical FEF calculated for SS samples, SS1190 (a), SS620 (b), SS239 (c), and SS96 (d).

spectra are larger than those in the experimental spectra by a factor of approximately 1.3. The good agreement between the experimental and theoretical FLEF line shapes confirms again that the experimental FLEF spectra reflect well the integral behaviors of local electric fields inside the individual waveguide layers.

The FEF map of the sample SS1190 shown in Fig. 5(a) exhibits only a relatively broad distribution inside the WG1 layer corresponding to the excitation of the PWG1 mode and the electric fields inside the WG2 layer are too weak to be seen. In this sample, the S2 layer is so thick that the coupling between the PWG1 and PWG2 modes is too weak to excite the PWG2 mode. Reflecting the electric field distribution seen in Fig. 5(a), the Lorentzian peak appears only in the WG1 FLEF spectrum as seen in Figs. 4(a) and 4(e). As the thickness of the S2 layer decreases down to $620 \mathrm{~nm}$, the coupling strength becomes strong enough to excite the PWG2 mode and a very sharp field distribution is generated inside the WG2 layer at approximately $60.7^{\circ}$ as can be clearly seen in Fig. 5(b). At the same angle, the electric fields inside the WG1 layer are weakly suppressed; this is a situation very similar to that seen for the sample BS380 [Fig. 3(a)], although the amount of the suppression is very small. These behaviors of local electric fields lead to the strong and sharp Lorentzian peak in the WG2 FLEF spectra and the shallow dip inside the broader Lorentzian peak in the WG1 FLEF spectra [Figs. 4(b) and 4(f)].

As the S2 layer thickness decreases further, substantial changes in the electric field distributions take place as can be seen in Figs. 5(c) and 5(d). In fact, in both the WG1 and WG2 layers, the electric field distributions split into lowand high-angle parts and the separation between the two lobes becomes larger as the S2 thickness decreases. These changes in the electric field distributions are caused by the increase in the strength of the mode coupling and lead to double peaks in both the WG1 and WG2 FLEF spectra seen in Figs. 4(c), 4(d), 4(g), and 4(h). The splitting of the field distributions and the appearance of the FLEF double peaks for the samples SS239 and SS96 are thought to be due to the formation of the coupled modes in the strong coupling regime $[27,30]$. In our SS239 and SS96 samples, a symmetric and an antisymmetric coupled mode are expected to be formed by in-phase and out-of-phase superpositions of the PWG1 and PWG2 modes, respectively. The nature of the modes can be well documented by examining the electric field distributions seen in Figs. 5(c) and 5(d) along the $z$ direction; in Fig. S4 of the Supplemental Material [49], plots of FEF versus $z$ are given for the lowangle and high-angle modes of the sample SS96. In fact, we see that for the low-angle mode the electric field inside the S2 layer (coupling layer) goes down to zero around its center, allowing us to assign this mode to the antisymmetric mode generated by the out-of-phase superposition of the evanescent tails inside the coupling layer. On the other hand, the high-angle mode can be assigned to the symmetric mode, in which the evanescent fields are superposed in-phase and the electric fields have finite values inside the coupling layer.

The results of fluorescence measurements and the analyses based on the EM calculations presented in this section indicate that the line shapes of experimental WG1 and WG2 FLEF spectra reproduce well the integral behaviors of local electric fields inside the WG1 and WG2 layers. The presently proposed technique of the fluorescence measurements, i.e., doping two different types of fluorophores separately into two waveguide layers and monitoring separately their fluorescence intensities at different wavelengths, is thus successful to trace experimentally the different behaviors of local electric fields 
inside the two waveguide layers. In the present studies, although it is necessary to choose carefully the fluorophores and wavelengths of fluorescence detections, the method used for preparing the fluorophore-doped waveguide layers, i.e., spin coating of mixed solutions of fluorophores and polymers, is very common and has been used widely. Therefore, it is straightforward to extend the present technique of probing the local electric fields to similar structures composed of other polymers and other fluorophores. Since a variety of techniques to prepare waveguides made of inorganic materials such as $\mathrm{Si}$ and $\mathrm{SiO}_{2}$ doped with various light emitters (fluorescent molecules, quantum dots, rare-earth ions, and so on) have also been well developed, the present technique is expected to be applicable also to inorganic planar waveguides. Furthermore, the presently proposed technique is thought to be extendable to more complex structures, including the stripe waveguides and diffraction gratings, when the doping techniques are combined with highly developed modern nanofabrication techniques such as e-beam lithography, photolithography, as well as chemical processes. The present technique is expected to be successful, when the fluorescence excitation spectra are measured with carefully chosen light emitters under resonant excitation and nonresonant emission conditions.

For both the BS and SS series of samples, the present experimental FLEF spectra clearly demonstrate that the local electric fields in the WG1 and WG2 layers change in quite different ways depending on the coupling strength, which are usually only speculated based on the EM calculations. We also note that the present samples give rise to the fluorescence enhancement. As can be seen in Figs. 2 and 4, the maximum enhancement factor obtained experimentally is as high as 89 [WG2 FLEF spectrum in Fig. 4(b)]. The strong dependence of the WG1 and WG2 FLEF spectra and their relative magnitudes on the excitation angle shown here can be used to mix the DCM and C314 fluorescence spectra with different weights and develop light sources with adjustable color hue. When a sharp Fano line shape is combined, large colorimetric changes are expected and may allow highly sensitive angle sensors and refractive index sensors to be realized.

\section{ANALYSES BASED ON COUPLED OSCILLATOR MODEL}

\section{A. Coupled oscillator model}

As demonstrated in the previous section, the behaviors of local electric fields inside the WG1 and WG2 layers are greatly modified by changing the S2 layer thickness, i.e., the strength of mode coupling. Although the experimental FLEF spectra can be well reproduced by the EM calculations, the mechanism of observed changes in the line shape is not well understood only by the results of EM calculation. For example, the change in the thickness of the S2 spacer layer may alter not only the strength of coupling between the PWG1 and PWG2 modes but also the resonance positions and widths of the modes. However, the EM results show both effects at the same time and do not allow us to separate them. A simple mechanical model, coupled oscillator (CO) model, has widely been used to discuss optical responses of various nanostructures including metallic and dielectric nanostructures [3,5,27,30,44,54-56]. In our previous paper [19], we have demonstrated that the $\mathrm{CO}$ model can describe fairly well the Fano resonances observed in metal-dielectric multilayer systems, in which the coupling between a SPP and a PWG mode plays a crucial role.

As schematically shown in Fig. 6(a), we consider a system of two oscillators (OCL1 and OCL2) connected by a spring and assume that only the OCL1 is driven by an external force. The equations of motion for the two oscillators can be written as [55]

$$
\begin{gathered}
\frac{d^{2} x_{1}}{d t^{2}}+\gamma_{1} \frac{d x_{1}}{d t}+\omega_{1}^{2} x_{1}+g x_{2}=f_{0} e^{i \omega t}, \\
\frac{d^{2} x_{2}}{d t^{2}}+\gamma_{2} \frac{d x_{2}}{d t}+\omega_{2}^{2} x_{2}+g x_{1}=0,
\end{gathered}
$$

where $x_{1}$ and $x_{2}$ stand for the displacements of OCL1 and OCL2, $\gamma_{1}$ and $\gamma_{2}$ are the damping constants, and $\omega_{1}$ and $\omega_{2}$ are the resonance frequencies, respectively. $f_{0} e^{i \omega t}$ is the external force acting on the OCL1. $g$ is the coupling constant. Assuming harmonic oscillations for $x_{1}$ and $x_{2}$ written as $x_{n}=A_{n} e^{i \omega t}(n=1,2)$ and inserting into Eqs. (3) and (4), we can obtain explicit expressions of amplitudes $A_{1}$ and $A_{2}$ in the steady state condition as

$$
\begin{aligned}
& \frac{A_{1}}{f_{0}}=\frac{\omega_{2}^{2}+i \gamma_{2} \omega-\omega^{2}}{\left(\omega_{1}^{2}+i \gamma_{1} \omega-\omega^{2}\right)\left(\omega_{2}^{2}+i \gamma_{2} \omega-\omega^{2}\right)-g^{2}}, \\
& \frac{A_{2}}{f_{0}}=\frac{-g}{\left(\omega_{1}^{2}+i \gamma_{1} \omega-\omega^{2}\right)\left(\omega_{2}^{2}+i \gamma_{2} \omega-\omega^{2}\right)-g^{2}} .
\end{aligned}
$$

Equations (5) and (6) allow us to calculate the $\omega$ dependence of the amplitudes, which are usually used to describe optical responses, such as scattering, transmission and absorption, of photonic systems.

To apply the model to our multilayer structures, we correlate the OCL1 and OCL2 to the PWG1 and PWG2 modes as schematically shown in Fig. 6(b). In the Cartesian coordinate system shown in Figs. 1(a) and 6(b), local electric fields inside the $j$ th layer of the multilayer structure excited by a $s$-polarized plane wave incident from the prism have only the $y$ component and can generally be expressed as $E_{y}^{j}(x, z, t)=E_{j}(z) e^{i\left(k_{\|} x-\omega t\right)}$, where $k_{\|}$is the $x$ component of the wave vector (in-plane wave vector). To fulfill EM 

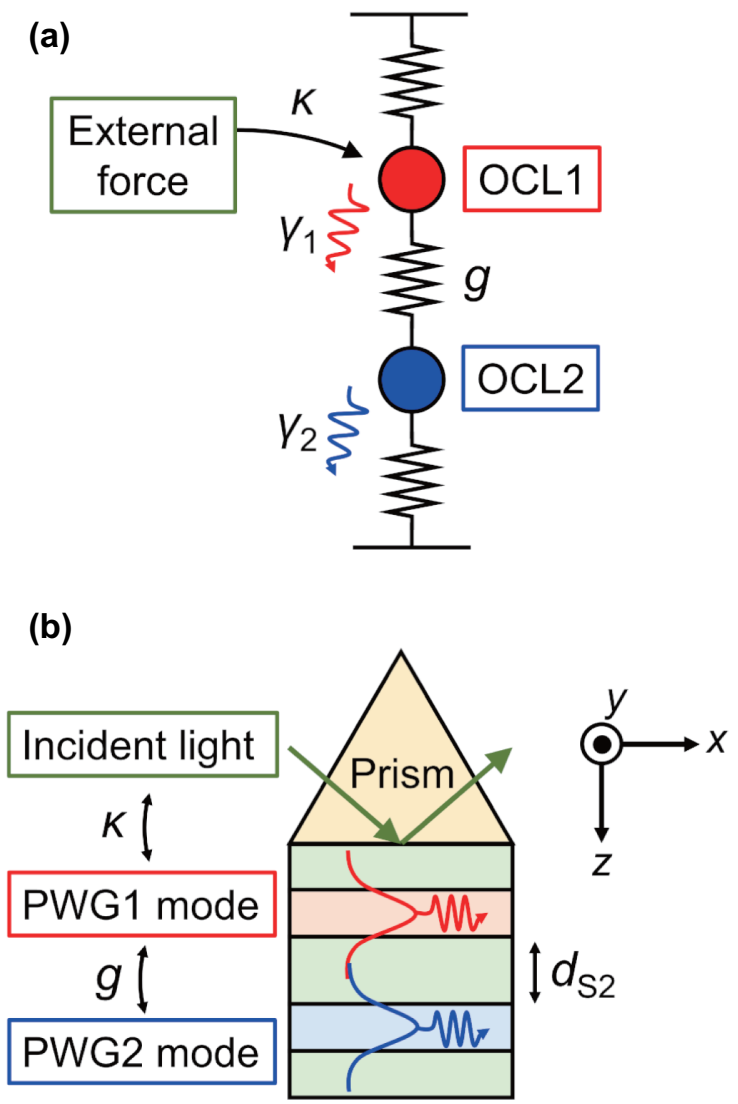

FIG. 6. Correspondence between coupled oscillator model and multilayer structure. (a) Coupled oscillator model describing a system of two harmonic oscillators OCL1 and OCL2 with damping constants of $\gamma_{1}$ and $\gamma_{2}$ connected by a spring with a coupling constant $g$. Only OCL1 couples to an external oscillation with a coupling constant $\kappa$. (b) Multilayer structure in which PWG1 and PWG2 modes are coupled with a coupling constant $g$. Incident light excite only PWG1 mode with a coupling strength $\kappa$.

boundary conditions at the interfaces, $k_{\|}$and $\omega$ must be identical to those of the excitation light. In the present experiments performed with the angle-scan ATR excitation, the wavelength of the excitation light $\lambda_{\text {ex }}$ is fixed, while the angle of incidence $\theta_{\text {in }}$ is varied. This means that $\omega$ is fixed, and $k_{\|}$varies as $k_{\|}=k_{0} n_{\mathrm{p}} \sin \theta_{\text {in }}$, where $n_{\mathrm{p}}$ is the refractive index of the prism and $k_{0}=2 \pi / \lambda_{\text {in }}$. As demonstrated in the previous section, the observed FLEF spectra reflect the variation of the local electric fields inside the WG1 and WG2 layers as a function of $k_{\|}$, not as a function of $\omega$. The first modification imposed on Eqs. (5) and (6) is the replacement of the variable $t$ by $x$ to describe the spatial variation rather than the temporal variation. Accordingly, $\omega$ must be replaced by $k_{\|}$. In the following discussions, we omit the factor $e^{-i \omega t}$ in the expression of $E_{y}^{j}(x, z, t)$, since $\omega$ is a constant, and we are interested in the absolute values of the local electric fields.

In the present ATR geometry, the PWG1 mode is not excited directly by the incident wave in the prism but excited by an evanescent field penetrating the S1 layer under the total reflection condition. Therefore, we introduce a coupling constant $\kappa$ that connects the incident field inside the prism to the field exciting the PWG1 mode; we replace the external force $f_{0} e^{i \omega t}$ in Eq. (3) by the excitation field $\kappa E_{0} e^{i k_{\|} x}$, where $E_{0}$ is the amplitude of incident field inside the prism. Writing the local electric fields at $z=z_{1}$ in the WG1 layer and at $z=z_{2}$ in the WG2 layer as $E_{\mathrm{WG} n}(x)=E_{n}\left(z_{n}\right) e^{i k_{\|} x}(n=1,2)$ and correlating them to $x_{1}$ and $x_{2}$ of OCL1 and OCL2, we can obtain the following counter parts of Eqs. (5) and (6) that allow us to analyze the EM responses of our multilayer systems in the spirit of the CO model,

$$
\begin{aligned}
& \frac{E_{1}\left(z_{1}\right)}{E_{0}}=\frac{\left(k_{2}^{2}+i \gamma_{2} k_{\|}-k_{\|}^{2}\right) \kappa}{\left(k_{1}^{2}+i \gamma_{1} k_{\|}-k_{\|}^{2}\right)\left(k_{2}^{2}+i \gamma_{2} k_{\|}-k_{\|}^{2}\right)-g^{2}}, \\
& \frac{E_{2}\left(z_{2}\right)}{E_{0}}=\frac{-g \kappa}{\left(k_{1}^{2}+i \gamma_{1} k_{\|}-k_{\|}^{2}\right)\left(k_{2}^{2}+i \gamma_{2} k_{\|}-k_{\|}^{2}\right)-g^{2}},
\end{aligned}
$$

where $k_{1}$ and $k_{2}$ represent the in-plane wave vectors of the PWG1 and PWG2 modes, respectively. Within the modified CO model, Eqs. (7) and (8) allow us to calculate very easily FEF defined as $\left|E_{1}\left(z_{1}\right) / E_{0}\right|^{2}$ and $\left|E_{2}\left(z_{2}\right) / E_{0}\right|^{2}$.

Using Eqs. (7) and (8) we can analyze the $k_{\|}$dependence of FEF at various positions in the WG1 and WG2 layers. However, as discussed in detail in the previous section, the experimental FLEF spectra reflect the behaviors of the FEF integrated over the WG1 and WG2 layers. Therefore, to analyze the integrated FEF, it is necessary to further simplify the model. For convenience, we use averages of FEF for the WG1 and WG2 layers, which can be obtained by dividing the integrated FEF by the layer thicknesses. We assume that the parameters of the $\mathrm{CO}$ model appearing in Eqs. (7) and (8) $\left(k_{1}, k_{2}, \gamma_{1}, \gamma_{2}\right.$, and $\left.g\right)$ are independent of $z_{1}$ and $z_{2}$ and write the right-hand sides of Eqs. (7) and (8) as $R 1\left(k_{\|}\right)$and $R 2\left(k_{\|}\right)$, respectively. Then, the averages of FEF for the WG1 and WG2 layers, written as $\overline{\mathrm{FEF} 1}$ and $\overline{\mathrm{FEF}}$, are simply given by $\overline{\mathrm{FEF} 1}=\left|R 1\left(k_{\|}\right)\right|^{2}$ and $\overline{\mathrm{FEF} 2}=\left|R 2\left(k_{\|}\right)\right|^{2}$, respectively. These are the basic equations used in the following analyses.

In Eqs. (7) and (8), $g$ is merely a constant that gives the strength of coupling between the PWG1 and PWG2 modes. However, as presented in Sec. III, the main concern of the present experiments and EM analyses is to investigate the changes of EM responses caused by the change in the coupling strength, which is governed by the S2 layer thickness $d_{\mathrm{S} 2}$. Therefore, to perform a systematic analysis of the EM results based on the modified $\mathrm{CO}$ model, we introduce a phenomenological expression of $g$ that describes the dependence on $d_{\mathrm{S} 2}$. For our multilayer structures, the PWG1 and PWG2 modes couple with each other through the overlap of evanescent 


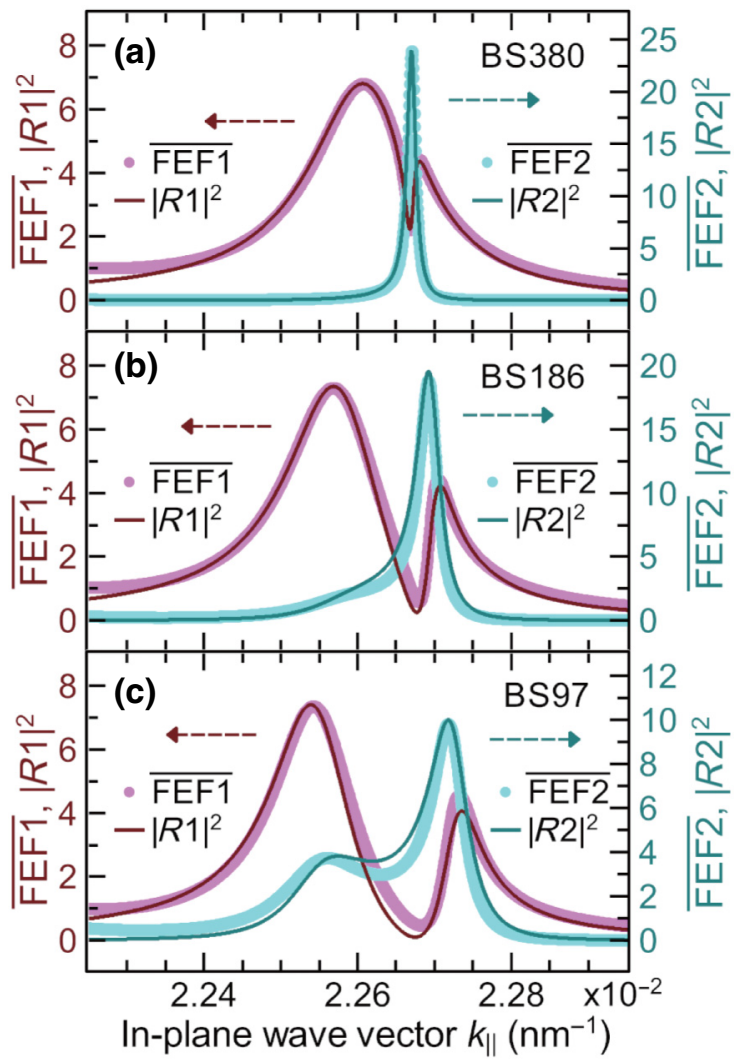

FIG. 7. Spectra of field enhancement factor averaged over WG1 ( $\overline{\mathrm{FEF} 1})$ and WG2 ( $\overline{\mathrm{FEF}}$ ) layers and results of fitting to $|R 1|^{2}$ and $|R 2|^{2}$ of coupled oscillator model for BS samples, BS380 (a), BS186 (b), and BS97 (c).

fields associated with the modes that penetrate the S2 spacer layer. Since the evanescent fields decay exponentially away from the WG1-S2 and S2-WG2 interfaces, the strength of the coupling between the PWG1 and PWG2 modes is expected to decrease exponentially as $d_{\mathrm{S} 2}$ increases. Taking this mechanism of coupling into account, we introduce phenomenologically a function written as $g=g_{0} \exp \left(-\left|k_{\perp}^{\mathrm{S} 2}\right| d_{\mathrm{S} 2}\right)$, where $g_{0}$ is a constant and $k_{\perp}^{\mathrm{S} 2}$ is the $z$ component of the wave vector (out-of-plane wave vector) inside the $\mathrm{S} 2$ layer given by $k_{\perp}^{\mathrm{S} 2}=\sqrt{\left(k_{0} n_{\mathrm{S} 2}\right)^{2}-k_{\|}^{2}}$ with $n_{\mathrm{S} 2}$ being the refractive index of the S2 layer. For $\kappa$, which describes the coupling between the incident light and the PWG1 mode via evanescent fields inside the S1 layer, in the same manner as $g$, we introduce $\kappa=\kappa_{0} \exp \left(-\left|k_{\perp}^{S 1}\right| d_{\mathrm{S} 1}\right)$, where $k_{\perp}^{\mathrm{S} 1}$ is given by $k_{\perp}^{\mathrm{S} 1}=\sqrt{\left(k_{0} n_{\mathrm{S} 1}\right)^{2}-k_{\|}^{2}}$ with the refractive index $n_{\mathrm{S} 1}$ of the $\mathrm{S} 1$ layer.

Examining closely the right-hand sides of Eqs. (7) and (8), we find that both equations have the same denominator $D\left(k_{\|}\right)=\left(k_{1}^{2}+i \gamma_{1} k_{\|}-k_{\|}^{2}\right)\left(k_{2}^{2}+i \gamma_{2} k_{\|}-k_{\|}^{2}\right)-g^{2}$. According to detailed studies of the $\mathrm{CO}$ system [27,55], it is known that normal modes (or eigenmodes) of the system are two coupled modes $(+$ and - modes) constructed by

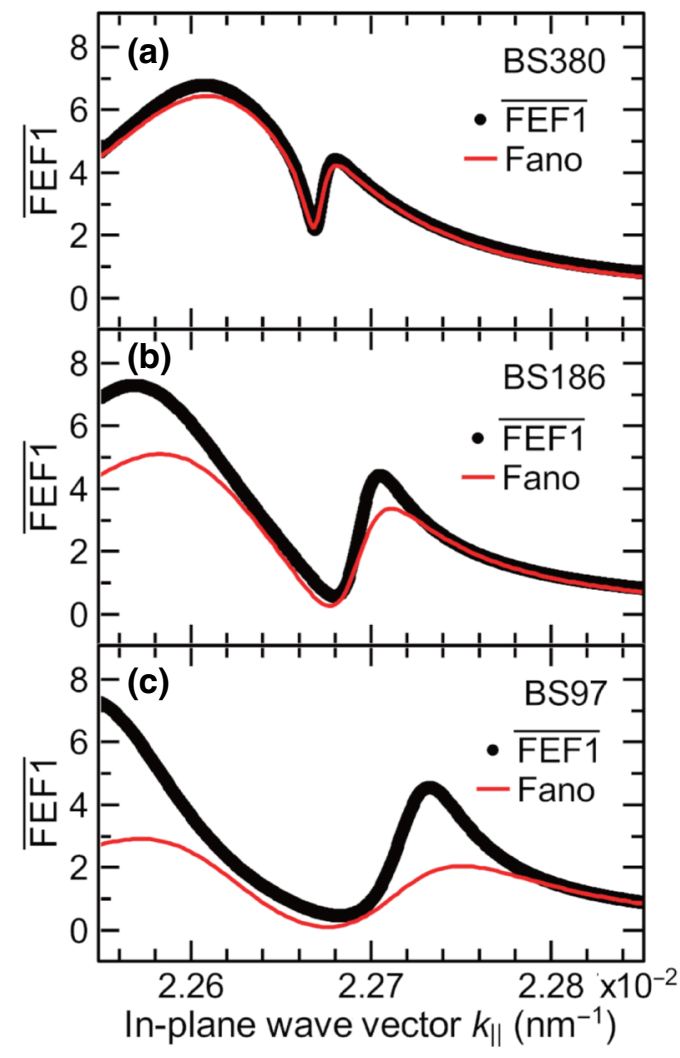

FIG. 8. Comparison of $\overline{\text { FEF1 }}$ spectra with line shapes calculated by generalized Fano function [Eq. (10)] for BS samples, BS380 (a), BS186 (b), and BS97 (c).

linear combinations of the uncoupled modes; these coupled modes are usually attributed to the symmetric and antisymmetric modes. In our modified $\mathrm{CO}$ model, $k_{\|}$values (eigenvalues) of the normal modes $\left(k_{\|}^{+}\right.$and $\left.k_{\|}^{-}\right)$can be obtained by solving $D\left(k_{\|}\right)=0$. When $\gamma_{1}=\gamma_{2}=0$ is assumed for simplicity, we can easily find eigenvalues $k_{\|}^{+}$ and $k_{\|}^{-}$that satisfy

$$
k_{\|}^{ \pm 2}=\frac{k_{1}^{2}+k_{2}^{2} \pm \sqrt{\left(k_{1}^{2}-k_{2}^{2}\right)^{2}+4 g^{ \pm 2}}}{2},
$$

where $g^{ \pm}$are $g$ values determined by $k_{\|}^{ \pm}$. From Eq. (9) it is easy to see that the mode splitting $\left(k_{\|}^{+}-k_{\|}^{-}\right)$increases as $g^{ \pm}$increases, i.e., as $d_{\mathrm{S} 2}$ decreases. Note that $k_{\|}^{+}$and $k_{\|}^{-}$ satisfying Eq. (9) take real values, when $\gamma_{1}$ and $\gamma_{2}$ are zero. However, for nonzero values of $\gamma_{1}$ and $\gamma_{2}, k_{\|}^{+}$and $k_{\|}^{-}$take complex values; when the values of $\gamma_{1}$ and $\gamma_{2}$ are small, the real parts of $k_{\|}^{+}$and $k_{\|}^{-}$representing the resonance positions are slightly shifted from those given by Eq. (9); inverses of nonzero imaginary parts of $k_{\|}^{+}$and $k_{\|}^{-}$give decay lengths of the normal modes along the $x$ direction.

In our analyses based on the modified $\mathrm{CO}$ model, we calculate $\overline{\mathrm{FEF} 1}=\left|R 1\left(k_{\|}\right)\right|^{2}$ and $\overline{\mathrm{FEF} 2}=\left|R 2\left(k_{\|}\right)\right|^{2}$ as 
functions of $k_{\|}$, where $k_{\|}$takes real values as in the experiments and EM analyses. Spectra of $\overline{\mathrm{FEF} 1}$ and $\overline{\mathrm{FEF} 2}$ are thus determined by the behavior of $1 /\left|D\left(k_{\|}\right)\right|^{2}$ as well as that of the numerators in $R 1\left(k_{\|}\right)$and $R 2\left(k_{\|}\right)$. In principle, $1 /\left|D\left(k_{\|}\right)\right|^{2}$ is expected to show two peaks at $\operatorname{Re}\left\{k_{\|}^{+}\right\}$ and $\operatorname{Re}\left\{k_{\|}^{-}\right\}$with widths and heights governed by $\gamma_{1}$ and $\gamma_{2}$ values. Differences between the spectra of $\overline{\mathrm{FEF} 1}$ and FEF2 are brought by different behaviors of the numerators in $R 1\left(k_{\|}\right)$and $R 2\left(k_{\|}\right)$. A variety of spectra are generated by the modified $\mathrm{CO}$ model by various sets of the oscillator parameters.

\section{B. Analyses of BS sample series}

First, we calculate the averages of FEF inside the WG1 and WG2 layers, $\overline{\text { FEF1 }}$ and $\overline{\text { FEF2}}$, as functions of $k_{\|}$, using the structural parameters of the BS380, BS186, and BS97 samples (Table S2 within the Supplemental Material [49]), and then fit them to $\left|R 1\left(k_{\|}\right)\right|^{2}$ and $\left|R 2\left(k_{\|}\right)\right|^{2}$ to find parameters of the two oscillators, where $R 1\left(k_{\|}\right)$and $R 2\left(k_{\|}\right)$represent the right-hand sides of Eqs. (7) and (8), respectively. In Fig. 7, the spectra of $\overline{\text { FEF1 }}$ and $\overline{\text { FEF2 }}$ are plotted together with the fit curves. The oscillator parameters obtained are listed in Table S5 of the Supplemental Material [49]. Figure 7 shows that although deviations of the fit curves from the spectra of $\overline{\mathrm{FEF} 1}$ and $\overline{\mathrm{FEF}} 2$ become a little more noticeable for the sample BS97, they reproduce very well the overall changes in the line shape of the $\overline{\mathrm{FEF} 1}$ and $\overline{\mathrm{FEF} 2}$ spectra. It should be noted that in the fitting procedures, the prefactor $g_{0}$ in the expression of $g$ is assumed to be a constant $\left(g_{0}=6.75 \times 10^{-6} \mathrm{~nm}^{-2}\right)$ for different samples with different thicknesses $\left(d_{\mathrm{S} 2}=380,186\right.$, and $\left.97 \mathrm{~nm}\right)$. The results of successful fitting seen in Fig. 7 assure the validity of the functional form of $g$ phenomenologically introduced in the preceding section.

As pointed out in Sec. III A, the spectrum of $\overline{\text { FEF1 }}$ for the sample BS380 exhibits a Fano line shape and the line shape is modified as the coupling strength increases. Although detailed analyses of the Fano line shape is not possible by the EM theory only, the CO model provides a tool to gain insight into the changes in the asymmetric line shape. In fact, under an assumption of $\gamma_{1} \gg \gamma_{2}$, i.e., in the case of coupling between a broad mode and a sharp mode, and when a function defined by $\mathcal{L}=k_{1}^{2}+i \gamma_{1} k_{\|}-k_{\|}^{2}$ is slowly varying around the sharp mode at $k_{\|}=k_{2}$, Gallinet [56] derived an approximate form of $\left|R 1\left(k_{\|}\right)\right|^{2}$ written as

$$
\left|R 1\left(k_{\|}\right)\right|^{2} \simeq \frac{\kappa^{2}}{|\mathcal{L}|^{2}} \frac{(s+q)^{2}+b}{s^{2}+1} .
$$

The first term of the right-hand side of Eq. (10) describes a Lorentzian line shape generated by the broad mode at $k_{\|}=k_{1}$. The second term is a Fano function that describes the asymmetric Fano line shape, where $s$ is a variable given by

$$
s=\frac{k_{\|}^{2}-k_{2}^{2}+k_{2} \Delta}{\Gamma},
$$

with a resonance shift $\Delta$ and a line width $\Gamma$, and $q$ and $b$ are an asymmetry parameter and a modulation damping parameter, respectively. The parameters in the Fano function are connected to the oscillator parameters as summarized within the Supplemental Material [49]. Gallinet et al. $[42,57]$ have shown that Fano line shapes of a variety of Fano-resonant nanostructures can be well described by Eq. (10). Since Eq. (10) is a generalization of the Fano function to the case of oscillators with intrinsic losses, the right-hand side of Eq. (10) is hereafter referred to as the generalized Fano function.

Since we know the oscillator parameters for the BS380, BS186, and BS97 samples (Table S5 in the Supplemental Material [49]), it is straightforward to convert the parameters to those of the generalized Fano function and plot the resulting line shapes. In Figs. 8(a)-8(c), spectra of $\overline{\mathrm{FEF} 1}$ for the three different samples [the same as those in Figs. 7(a)-7(c)] are compared with line shapes generated by the generalized Fano function in a narrow region around the asymmetric line shape. We see that for the sample BS380, the asymmetric line shape of $\overline{\mathrm{FEF} 1}$ near $k_{\|}=k_{2}$ can be reproduced almost perfectly by the generalized Fano function; this allows us to finally conclude that the asymmetric line shape observed for the sample BS380 indeed stems from the Fano resonance. For the samples BS186 and BS97 with larger coupling strengths (thinner

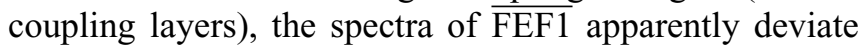
from the line shapes generated by the generalized Fano function around both the asymmetric part near $k_{\|}=k_{2}$ and the symmetric part near $k_{\|}=k_{1}$; the deviation becomes larger as the coupling strength increases. The deviation of the line shape implies that the approximation leading to Eq. (10) is no more valid in the case of larger coupling strengths. The application of the generalized Fano function (an approximate form of the $\mathrm{CO}$ response) allows us to conclude more clearly the deviation of the line shape from the Fano line shape already suggested in Sec. III A.

Based on the modified $\mathrm{CO}$ model, we can explain the line shape changes as follows. When the coupling between the PWG1 and PWG2 modes is weak enough, characteristics of the uncoupled modes such as the resonance positions and widths are still conserved without large changes but a part of energy of the PWG1 mode is transferred to the sharp PWG2 mode due to the coupling, resulting in a sharp Fano dip in the broad response of the

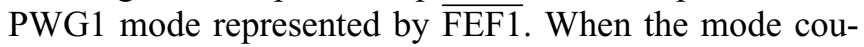
pling becomes stronger, the characteristics of the uncoupled modes start to be lost and the modes excited in the system tend to be the coupled modes characterized by the eigenvalues $k_{\|}^{+}$and $k_{\|}^{-}$. When the coupling is strong 
enough, both the $\overline{\mathrm{FEF} 1}$ and $\overline{\mathrm{FEF} 2}$ spectra exhibit two peaks corresponding to $k_{\|}^{+}$and $k_{\|}^{-}$as demonstrated in the following section for the SS sample series. The strengths of coupling for the samples BS186 and BS97 are not so strong to generate two well-separated peaks but fall into an intermediate region where the line shape makes a transition from the Fano to double-peak shape. The appearance and growth of the shoulder at lower values of $k_{\|}$in the spectra of $\overline{\text { FEF2 }}$ for the samples BS186 and BS97 [Figs. 7(b) and 7(c)] are signs of the initial stage of the formation of the coupled modes. Therefore, the asymmetric dips seen in the corresponding spectra of $\overline{\mathrm{FEF} 1}$ reflect not only the energy transfer from the PWG1 mode to the PWG2 mode but also the emergence of double peaks. As the coupling strength increases, the dip tends to be a valley between the two peaks rather than a Fano dip; this type of line shape is often attributed to the classical analog of Autler-Townes splitting $[14,39,40]$.

\section{Analyses of SS sample series}

As described in Sec. III B, the experimental results for the SS sample series cover a wide range of the coupling strength. However, examining closely the structural parameters of the SS samples listed in Table S3 in the Supplemental Material [49], we find that along with the systematic change in the $\mathrm{S} 2$ spacer thickness $d_{\mathrm{S} 2}$, other parameters (thicknesses and refractive indices) fluctuate to some extent from sample to sample due to experimental errors. To discuss the effects caused purely by the change in $d_{\mathrm{S} 2}$ it is desirable that all the parameters except for $d_{\mathrm{S} 2}$ are fixed. For this reason, in the following discussion, we assume ideal samples, in which only $d_{\mathrm{S} 2}$ takes different values and other structural parameters are fixed; for the ideal samples, except for $d_{\mathrm{S} 2}$, we use values of the structural parameters averaged over four different SS samples. The structural parameters for the ideal samples are listed in Table S7 of the Supplemental Material [49]. Figures 9(a)-9(e) show spectra of $\overline{\mathrm{FEF} 1}$ and $\overline{\mathrm{FEF} 2}$ calculated for the ideal samples with $d_{\mathrm{S} 2}=650,470,310,200$, and $100 \mathrm{~nm}$; hereafter, using the thickness values, we call the ideal samples ISS650, ISS470, ISS310, ISS200, and ISS100, respectively. In the figures, we see very clearly the systematic change in the line shape depending on $d_{\mathrm{S} 2}$ very similar to that seen in Figs. 4(b)-4(d) for the samples SS620, SS239, and SS96.

We calculate the spectra of $\overline{\mathrm{FEF} 1}$ and $\overline{\mathrm{FEF} 2}$ by varying continuously $d_{\mathrm{S} 2}$ in a very wide range with a step of $10 \mathrm{~nm}$. The results are presented as color maps in Figs. 9(f) and $9(\mathrm{~g})$ for $\overline{\mathrm{FEF} 1}$ and $\overline{\mathrm{FEF}}$, respectively. In these figures, we see that when $d_{\mathrm{S} 2}$ is larger than approximately $800 \mathrm{~nm}$, only the local electric field generated inside the WG1 layer is visible, and the local field in the WG2 layer is too weak to be seen. In this region of $d_{\mathrm{S} 2}$, the mode coupling is too weak to excite efficiently the PWG2 mode. When $d_{\mathrm{S} 2}$ falls into an intermediate region $\left(400<d_{\mathrm{S} 2}<800 \mathrm{~nm}\right)$, the coupling strength becomes large enough to excite the PWG2 mode and consequently, the $\overline{\text { FEF2 }}$ spectrum exhibits a peak similar to that in Fig. 9(a), while the $\overline{\text { FEF1 }}$ spectrum exhibits a dip due to the suppression of electric field caused by the energy transfer to the PWG2 mode. In this intermediate region, changes in the line shapes in the spectra of $\overline{\mathrm{FEF} 1}$ and $\overline{\mathrm{FEF} 2}$ caused by the decrease in $d_{\mathrm{S} 2}$ are very similar to those seen for the BS samples discussed in the previous section. This intermediate region of $d_{\mathrm{S} 2}$ is a transition region from the weak to strong coupling. For $d_{\mathrm{S} 2}$ smaller than approximately $400 \mathrm{~nm}$, Figs. 9(f) and 9(g) show clearly the upper and lower branches corresponding to the double peaks in both the $\overline{\mathrm{FEF} 1}$ and $\overline{\mathrm{FEF} 2}$ spectra [Figs. 9(d) and 9(e)]; the mode splitting increases continuously as $d_{\mathrm{S} 2}$ decreases, a feature typical in the strong coupling regime.

The results presented in Fig. 9 give us suggestions about the enhancement of local electric fields. As can be seen in

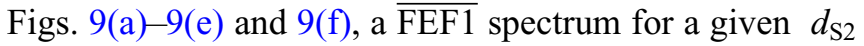
value takes a peak value at the low- $k_{\|}$peak [lower branch in Fig. 9(f)]. The peak value (approximately 30) does not depend strongly on $d_{\mathrm{S} 2}$. On the other hand, the peak value in the $\overline{\text { FEF} 2 ~ s p e c t r u m ~ d e p e n d s ~ s t r o n g l y ~ o n ~} d_{\mathrm{S} 2}$. Figure $9(\mathrm{~g})$ shows that there exists an optimum $\mathrm{S} 2$ thickness, i.e., $d_{\mathrm{S} 2}=470 \mathrm{~nm}$, that leads to the maximum value of approximately 50 in the high- $k_{\|}$branch. We note that this behavior of the local field enhancement in the WG2 layer is very similar to that predicted by Gallinet et al. [39] for Fanoresonant plasmonic nanostructures from EM calculations performed with a surface integral equations method. They studied the optical response of a gold nanorod antenna supporting a dipolar radiative mode coupled to two parallel nanorods supporting a nonradiative quadrupolar mode; the strength of coupling can be controlled by the offset of the dipolar nanorod antenna from the symmetric position. They calculated the local electric field enhancement associated with the excitation of the quadrupolar mode and reported its dependence on the coupling strength very similar to that of our $\overline{\text { FEF2 }}$ seen in Fig. 9(g). They argued that the maximum field enhancement is achieved under the critical coupling condition, where the intrinsic loss of the quadrupolar mode balances its radiative loss; the intrinsic loss stems from the power dissipation in the parallel nanorods (nonradiative loss) and the radiative loss is determined by the recoupling of the nonradiative quadrupolar mode to the radiative dipolar mode. It is interesting to note that in both our dielectric multilayer structure and their plasmonic nanostructure, the critical coupling takes place for a coupling strength falling in the transition region from the weak to strong coupling regime. The enhanced local electric fields inside the WG2 layer can be applied to enhance optical signals such as Raman scattering and second-harmonic generation. The results presented above suggest that to develop potential applications using 

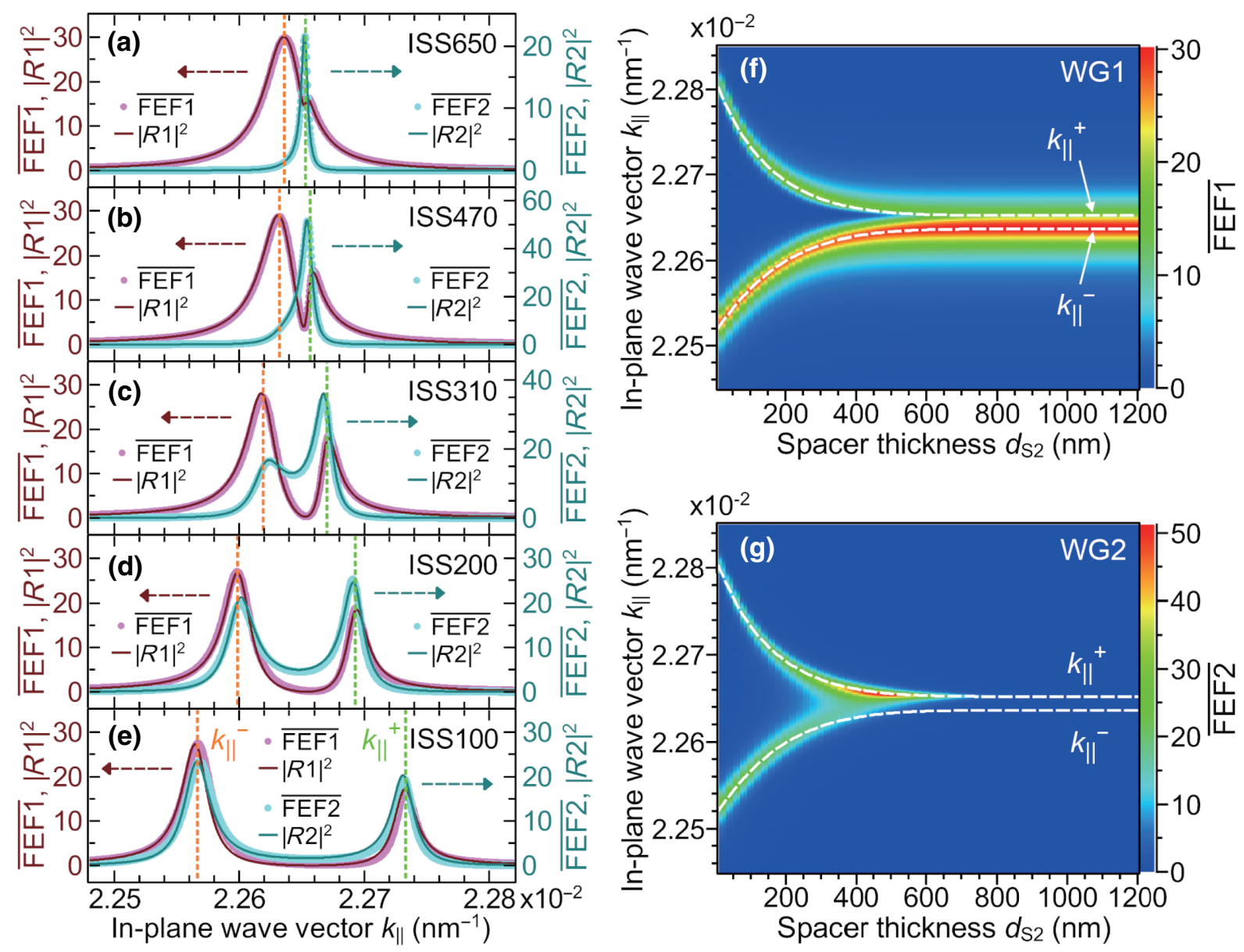

FIG. 9. Spectra of field enhancement factor averaged over WG1 $(\overline{\mathrm{FEF} 1})$ and WG2 $(\overline{\mathrm{FEF}})$ layers and results of fitting to $|R 1|^{2}$ and $|R 2|^{2}$ of coupled oscillator model for ideal SS samples, ISS650 (a), ISS470 (b), ISS310 (c), ISS200 (d), and ISS100 (e). Vertical dashed lines are positions of eigenvalues $k_{\|}^{+}$and $k_{\|}^{-}$. Maps of $\overline{\text { FEF1 }}$ (f) and $\overline{\text { FEF2 }}$ (g) of the ISS sample obtained by varying spacer thickness $d_{\mathrm{S} 2}$ in a wide range. White dashed lines are positions of eigenvalues $k_{\|}^{+}$and $k_{\|}^{-}$.

analogous multilayer structures, it is necessary to achieve the critical coupling condition by optimizing the structural parameters.

We now proceed to analyze the EM results of the ISS samples by applying the modified CO model. We fit the $\overline{\text { FEF1 }}$ and $\overline{\text { FEF2 }}$ spectra of the ISS samples to CO responses $\left|R 1\left(k_{\|}\right)\right|^{2}$ and $\left|R 2\left(k_{\|}\right)\right|^{2}$. The fit curves obtained are presented by solid lines in Figs. 9(a)-9(e). We see that the FEF1 and $\overline{\text { FEF} 2}$ spectra are very well reproduced by the fit curves in a wide range of $d_{\mathrm{S} 2}$. It should be noted that the good fit could be obtained by setting the prefactor of the coupling constant $g_{0}$ to the same value as that used for the BS samples, i.e., $g_{0}=6.75 \times 10^{-6} \mathrm{~nm}^{-2}$. The other fitting parameters, $k_{1}, k_{2}, \gamma_{1}$, and $\gamma_{2}$, are plotted in Figs. 10(a) and 10(b) as a function of $d_{\mathrm{S} 2}$. Figure 10(a) shows that the resonance wave numbers $k_{1}$ and $k_{2}$ of the uncoupled PWG1 and PWG2 modes shift to higher wave numbers as $d_{\mathrm{S} 2}$ decreases. On the other hand, Fig. 10(b) shows that as $d_{\mathrm{S} 2}$ decreases, $\gamma_{1}$ decreases, while $\gamma_{2}$ increases. The dotted lines in the figures show results of least-squares fits of the parameters to exponential functions; the functional form used and fitting parameters obtained are summarized within the Supplemental Material [49]. Figures 10(a) and 10(b) show that the variation of $d_{\mathrm{S} 2}$ in the multilayer structure leads to the changes in the characteristics (wave number and damping) of the uncoupled PWG1 and PWG2 modes in addition to the strength of mode coupling. In the results of EM calculations, the influences of $d_{\mathrm{S} 2}$ on the coupling strength and the mode characteristics appear at the same time and it is difficult to separate them. However, the above results indicate that the application of the $\mathrm{CO}$ model enables us to separate them.

Once the oscillator parameters are known, the eigenvalues (wave numbers) of the coupled modes can be obtained. Assuming $\gamma_{1}=\gamma_{2}=0$ and using values of $k_{1}$ and $k_{2}$ calculated from the exponential fit functions, we solve numerically $D\left(k_{\|}\right)=0$ to find $k_{\|}^{+}$and $k_{\|}^{-}$values by varying $d_{\mathrm{S} 2}$ with a step of $5 \mathrm{~nm}$, where $D\left(k_{\|}\right)$represents the 

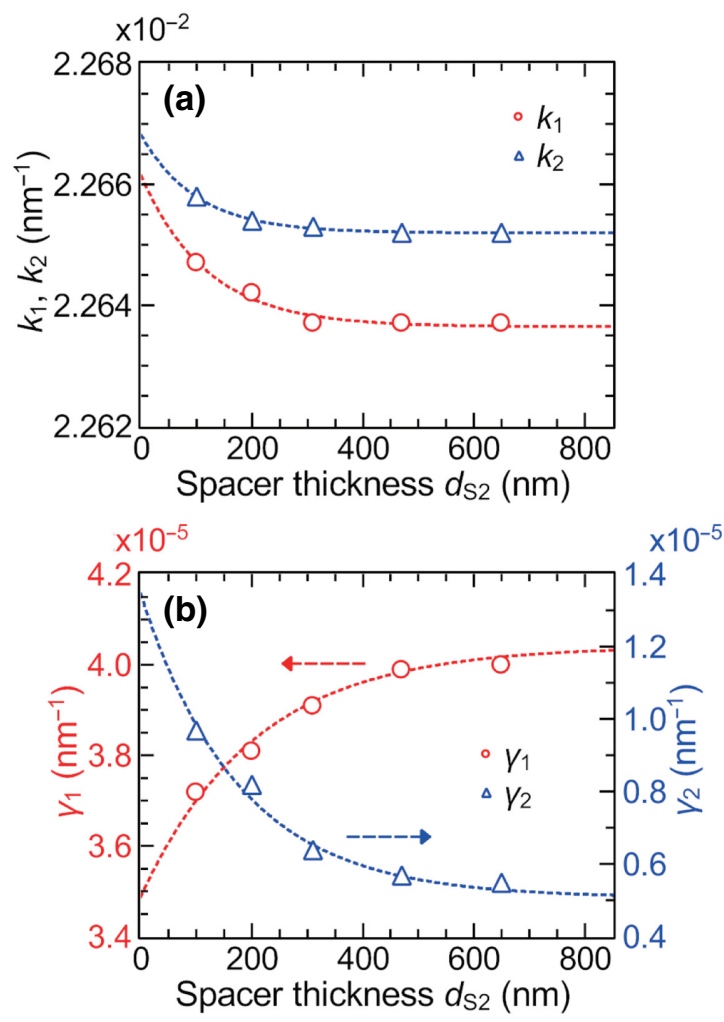

FIG. 10. Oscillator parameters for ideal samples plotted as a function of spacer thickness $d_{\mathrm{S} 2}$. In-plane wave vectors $k_{1}$ and $k_{2}$ (a), and damping constants $\gamma_{1}$ and $\gamma_{2}$ (b) of PWG1 and PWG2 modes. Dashed lines are results of least-squares fitting to exponential functions.

denominator of Eqs. (7) and (8). The dependencies of $k_{\|}^{+}$ and $k_{\|}^{-}$on $d_{\mathrm{S} 2}$ are plotted in Figs. 9(f) and 9(g) as dashed lines. The eigenvalues corresponding to the ISS samples are also shown by vertical dotted lines in Figs. 9(a)-9(e). A close examination of the figures reveals that in the region of $d_{\mathrm{S} 2}<300 \mathrm{~nm}$, where the coupling is strong enough to generate well-separated double peaks in both the $\overline{\text { FEF1 }}$ and FEF2 spectra, the positions of the double peaks are almost identical to $k_{\|}^{+}$and $k_{\|}^{-}$, indicating that the $\overline{\mathrm{FEF} 1}$ and $\overline{\mathrm{FEF} 2}$ spectra reflect almost faithfully the behavior of the coupled mode. On the other hand, as $d_{\mathrm{S} 2}$ increases in the region of $d_{\mathrm{S} 2}>300 \mathrm{~nm}$, the contribution of the $k_{\|}^{+}$mode in the $\overline{\mathrm{FEF} 1}$ spectrum and that of the $k_{\|}^{-}$mode in the $\overline{\mathrm{FEF} 2}$ spectrum tend to disappear and consequently, the $\overline{\mathrm{FEF} 1}$ and $\overline{\mathrm{FEF} 2}$ spectra do not correlate well with the behavior of the coupled modes. It is now clear that in the strong coupling regime, monitoring the spectra of local electric fields is effective to trace the behavior of the coupled modes. The analyses of the EM results of BS and SS sample series based on the $\mathrm{CO}$ model described above demonstrate that the behaviors of local electric fields can be well reproduced by simple analytical calculations and the model is very helpful to gain insight into physical origins of the changes in the local electric fields caused by the change in the coupling layer thickness.

\section{CONCLUSIONS}

Behaviors of local electric fields inside two dielectric waveguide layers interacting with each other in multilayer structures are studied by varying the coupling strength of waveguide modes. To study the behaviors of local electric fields in the respective waveguide layers separately, the layers are doped with two different fluorophores; the fluorophores are so chosen that they can be excited by the same excitation light and their fluorescence can be monitored separately using two different detection wavelengths. Exciting the multilayer samples in the ATR configuration, angle-scan fluorescence excitation spectra are measured and normalized to the fluoresce intensity of the reference sample to obtain fluorescence enhancement spectra. The experimental fluorescence enhancement spectra measured for two different sample series agree well with theoretical spectra calculated by the EM theory of an emitter embedded in the multilayer system. Since the theoretical fluorescence intensity for a waveguide layer is found to be proportional to the local field enhancement factor integrated over the waveguide layer, the good agreement between experiment and theory assures that the experimental fluorescence enhancement spectra indeed reflect well the integral behaviors of the local electric fields inside the waveguide layers. Normally, the behaviors of local electric fields are difficult to observe experimentally and only speculated based on EM calculations. However, the present technique of fluorophore doping offers a simple way to visualize the local electric fields and is not difficult to extend to similar multilayer structures as well as various nanostructures.

In the BS series of samples in which a broad waveguide mode is coupled to a sharp waveguide mode, a Fano resonance and its modification caused by an increase in the coupling strength is demonstrated at the level of local electric fields. In the SS series of samples in which two sharp waveguide modes are coupled, the dependence of the local electric fields on the coupling strength is systematically studied in a wide range. In the strong coupling regime, the coupled-mode splitting is clearly observed in the spectra of local electric fields. A modified coupled oscillator model is applied to analyze the EM results. The simple analytical calculations based on the model can reproduce well the behaviors of the local electric fields, regardless the sample series and range of coupling strength. An approximate expression of the coupled oscillator model valid in the weak coupling regime is shown to be helpful to clarify the deviation of the line shape from the Fano line shape observed in the BS series of samples with increasing the coupling strength. For the SS series of samples, the analyses based on the coupled oscillator model reveal 
that the change in the thickness of the coupling layer leads to not only the change in the coupling strength but also the changes in the characteristics (wave number and damping) of the uncoupled waveguide modes. Furthermore, the analyses reveal that in the strong coupling regime, the spectra of the local electric fields inside the waveguide layers reflect almost faithfully the behaviors of the coupled modes. The present results demonstrate that the coupled oscillator model is helpful to gain these physical insights, which cannot be obtained by the EM results only.

\section{ACKNOWLEDGMENTS}

This work is supported by JSPS KAKENHI Grants No. 21J12954, No. 19K05307 and No. 16H03828. This work is also partially supported by Russian Foundation for Basic Research Grant No. 18-29-20006.

[1] B. Luk'Yanchuk, N. I. Zheludev, S. A. Maier, N. J. Halas, P. Nordlander, H. Giessen, and C. T. Chong, The Fano resonance in plasmonic nanostructures and metamaterials, Nat. Mater. 9, 707 (2010).

[2] A. E. Miroshnichenko, S. Flach, and Y. S. Kivshar, Fano resonances in nanoscale structures, Rev. Mod. Phys. 82, 2257 (2010).

[3] M. F. Limonov, M. V. Rybin, A. N. Poddubny, and Y. S. Kivshar, Fano resonances in photonics, Nat. Photonics 11, 543 (2017).

[4] Fano Resonances in Optics and Microwaves, edited by E. Kamenetskii, A. Sadreev, and A. Miroshnichenko (Springer Book, New York, 2018).

[5] B. Gallinet and O. J. F. Martin, Ab initio theory of Fano resonances in plasmonic nanostructures and metamaterials, Phys. Rev. B 83, 235427 (2011).

[6] U. Fano, Effects of configuration interaction on intensities and phase shifts, Phys. Rev. 124, 1866 (1961).

[7] M. Decker and I. Staude, Resonant dielectric nanostructures: a low-loss platform for functional nanophotonics, J. Opt. 18, 103001 (2016).

[8] C. Wu, A. B. Khanikaev, R. Adato, N. Arju, A. A. Yanik, H. Altug, and G. Shvets, Fano-resonant asymmetric metamaterials for ultrasensitive spectroscopy and identification of molecular monolayers, Nat. Mater. 11, 69 (2012).

[9] K. L. Lee, J. Bin Huang, J. W. Chang, S. H. Wu, and P. K. Wei, Ultrasensitive biosensors using enhanced Fano resonances in capped gold nanoslit arrays, Sci. Rep. 5, 8547 (2015).

[10] Y. Yu, M. Heuck, H. Hu, W. Xue, C. Peucheret, Y. Chen, L. K. Oxenløwe, K. Yvind, and J. Mørk, Fano resonance control in a photonic crystal structure and its application to ultrafast switching, Appl. Phys. Lett. 105, 061117 (2014).

[11] H. Grotewohl and M. Deutsch, Tunable geometric Fano resonances in a metal/insulator stack, J. Opt. 17, 085003 (2015).

[12] H. Grotewohl, B. Hake, and M. Deutsch, Intensity and phase sensitivities in metal/dielectric thin film systems exhibiting the coupling of surface plasmon and waveguide modes, Appl. Opt. 55, 8564 (2016).

[13] Y. Neo, T. Matsumoto, T. Watanabe, M. Tomita, and H. Mimura, Transformation from plasmon-induced transparence to -induced absorption through the control of coupling strength in metal-insulator-metal structure, Opt. Express 24, 26201 (2016).

[14] K. Matsunaga, Y. Hirai, Y. Neo, T. Matsumoto, and M. Tomita, Tailored plasmon-induced transparency in attenuated total reflection response in a metal-insulator-metal structure, Sci. Rep. 7, 17824 (2017).

[15] S. Hayashi, D. V. Nesterenko, and Z. Sekkat, Fano resonance and plasmon-induced transparency in waveguidecoupled surface plasmon resonance sensors, Appl. Phys. Express 8, 022201 (2015).

[16] S. Hayashi, D. V. Nesterenko, A. Rahmouni, and Z. Sekkat, Observation of Fano line shapes arising from coupling between surface plasmon polariton and waveguide modes, Appl. Phys. Lett. 108, 051101 (2016).

[17] Z. Sekkat, S. Hayashi, D. V. Nesterenko, A. Rahmouni, S. Refki, H. Ishitobi, Y. Inouye, and S. Kawata, Plasmonic coupled modes in metal-dielectric multilayer structures: Fano resonance and giant field enhancement, Opt. Express 24, 20080 (2016).

[18] S. Hayashi, Y. Fujiwara, B. Kang, M. Fujii, D. V. Nesterenko, and Z. Sekkat, Line shape engineering of sharp Fano resonance in Al-based metal-dielectric multilayer structure, J. Appl. Phys. 122, 163103 (2017).

[19] S. Hayashi, D. V. Nesterenko, and Z. Sekkat, Waveguidecoupled surface plasmon resonance sensor structures: Fano lineshape engineering for ultrahigh-resolution sensing, J. Phys. D: Appl. Phys. 48, 325303 (2015).

[20] D. V. Nesterenko, S. Hayashi, and Z. Sekkat, Extremely narrow resonances, giant sensitivity and field enhancement in low-loss waveguide sensors, J. Opt. 18, 065004 (2016).

[21] B. Kang, M. Fujii, D. V. Nesterenko, Z. Sekkat, and S. Hayashi, Fano resonances in near-field absorption in all-dielectric multilayer structures, J. Opt. 20, 125003 (2018).

[22] S. Hayashi, D. V. Nesterenko, A. Rahmouni, H. Ishitobi, Y. Inouye, S. Kawata, and Z. Sekkat, Light-tunable Fano resonance in metal-dielectric multilayer structures, Sci. Rep. 6, 33144 (2016).

[23] S. Hayashi, D. V. Nesterenko, A. Rahmouni, and Z. Sekkat, Polarization effects in light-tunable Fano resonance in metal-dielectric multilayer structures, Phys. Rev. B 95, 165402 (2017).

[24] S. Hayashi, D. V. Nesterenko, and Z. Sekkat, in LightTunable Fano Resonance in Metal-Dielectric Multilayer Structures, in Fano Resonances in Optics and Microwaves, edited by E. Kamenetskii, A. Sadreev, A. Miroshnichenko (Springer Book, New York, 2018), pp. 241-260.

[25] K. Motokura, B. Kang, M. Fujii, D. V. Nesterenko, Z. Sekkat, and S. Hayashi, Light-controllable Fano resonance in azo-dye-doped all-dielectric multilayer structure, J. Appl. Phys. 125, 223101 (2019).

[26] K. Motokura, B. Kang, M. Fujii, D. V. Nesterenko, Z. Sekkat, and S. Hayashi, Wide-range line shape control of Fano-like resonances in all-dielectric multilayer structures based on enhanced light absorption in 
photochromic waveguide layers, J. Appl. Phys. 127, 073103 (2020).

[27] L. Novotny, Strong coupling, energy splitting, and level crossings: A classical perspective, Am. J. Phys. 78, 1199 (2010).

[28] G. Khitrova, H. M. Gibbs, M. Kira, S. W. Koch, and A. Scherer, Vacuum Rabi splitting in semiconductors, Nat. Phys. 2, 81 (2006).

[29] The Physics of Semiconductor Microcavities: From Fundamentals to Nanoscale Devices, edited by B. Deveaud (Wiley-VCH Verlag GmbH \& Co. KGaA, Weinheim, 2006).

[30] P. Törmä and W. L. Barnes, Strong coupling between surface plasmon polaritons and emitters: a review, Rep. Prog. Phys. 78, 013901 (2015).

[31] D. G. Baranov, M. Wersäll, J. Cuadra, T. J. Antosiewicz, and T. Shegai, Novel nanostructures and materials for strong light-matter interactions, ACS Photonics 5, 24 (2018).

[32] D. S. Dovzhenko, S. V. Ryabchuk, Y. P. Rakovich, and I. R. Nabiev, Light-matter interaction in the strong coupling regime: configurations, conditions, and applications, Nanoscale 10, 3589 (2018).

[33] P. Vasa and C. Lienau, Strong light-matter interaction in quantum emitter/metal hybrid nanostructures, ACS Photonics 5, 2 (2018).

[34] C. Schneider, M. M. Glazov, T. Korn, S. Höfling, and B. Urbaszek, Two-dimensional semiconductors in the regime of strong light-matter coupling, Nat. Commun. 9, 2695 (2018).

[35] S. H. Autler and C. H. Townes, Stark effect in rapidly varying fields, Phys. Rev. 100, 703 (1955).

[36] S. Refki, S. Hayashi, A. Rahmouni, D. V. Nesterenko, and Z. Sekkat, Anticrossing behavior of surface plasmon polariton dispersions in metal-insulator-metal structures, Plasmonics 11, 433 (2016).

[37] T. Y. Abi-Salloum, Electromagnetically induced transparency and Autler-Townes splitting: Two similar but distinct phenomena in two categories of three-level atomic systems, Phys. Rev. A 81, 053836 (2010).

[38] P. M. Anisimov, J. P. Dowling, and B. C. Sanders, Objectively Discerning Autler-Townes Splitting from Electromagnetically Induced Transparency, Phys. Rev. Lett. 107, 163604 (2011).

[39] B. Gallinet, T. Siegfried, H. Sigg, P. Nordlander, and O. J. F. Martin, Plasmonic radiance: Probing structure at the Ångström scale with visible light, Nano Lett. 13, 497 (2013).

[40] B. Peng, Ş. K. Özdemir, W. Chen, F. Nori, and L. Yang, What is and what is not electromagnetically induced transparency in whispering-gallery microcavities, Nat. Commun. 5, 5082 (2014).

[41] H. Leng, B. Szychowski, M. C. Daniel, and M. Pelton, Strong coupling and induced transparency at room temperature with single quantum dots and gap plasmons, Nat. Commun. 9, 4012 (2018).

[42] B. Gallinet and O. J. F. Martin, Influence of electromagnetic interactions on the line shape of plasmonic Fano resonances, ACS Nano 5, 8999 (2011).
[43] B. Gallinet and O. J. F. Martin, Relation between near-field and far-field properties of plasmonic Fano resonances, Opt. Express 19, 22167 (2011).

[44] J. B. Lassiter, H. Sobhani, M. W. Knight, W. S. Mielczarek, P. Nordlander, and N. J. Halas, Designing and deconstructing the Fano lineshape in plasmonic nanoclusters, Nano Lett. 12, 1058 (2012).

[45] P. Alonso-Gonzalez, M. Schnell, P. Sarriugarte, H. Sobhani, C. Wu, N. Arju, A. Khanikaev, F. Golmar, P. Albella, L. Arzubiaga, F. Casanova, L. E. Hueso, P. Nordlander, G. Shvets, and R. Hillenbrand, Real-space mapping of Fano interference in plasmonic metamolecules, Nano Lett. 11, 3922 (2011).

[46] B. Kang, K. Motokura, M. Fujii, D. V. Nesterenko, Z. Sekkat, and S. Hayashi, Fano resonant behaviour of waveguide mode in all-dielectric multilayer structure directly monitored by fluorescence of embedded dye molecules, J. Opt. 21, 105006 (2019).

[47] B. Kang, K. Motokura, M. Fujii, D. V. Nesterenko, Z. Sekkat, and S. Hayashi, Observation of Fano line shape in directional fluorescence emission mediated by coupled planar waveguide modes and interpretation based on Lorentz reciprocity, AIP Adv. 10, 075302 (2020).

[48] M. N. Polyanskiy, "Refractive Index Database" (see https://refractiveindex.info, accessed 1 April 2021).

[49] See Supplemental Material at http://link.aps.org/supplemental/10.1103/PhysRevApplied.16.064065 for more information on sample fabrication, measured ATR spectra, and results of electromagnetic fitting, structural parameters of samples, consideration on the effect of emission function on fluorescence spectra, field distributions corresponding to the excitation of symmetric and antisymmetric modes, oscillator fit parameters, explicit expressions of Fano parameters in Fano approximation, exponential fit parameters for thickness dependence of oscillator parameters.

[50] RES-TEC, "Winspall Data Analysis Software" (see www.res-tec.de, accessed 1 April 2021).

[51] J. Enderlein, Single-molecule fluorescence near a metal layer, Chem. Phys. 247, 1 (1999).

[52] W. Lukosz, Light emission by multipole sources in thin layers. I. Radiation patterns of electric and magnetic dipoles, J. Opt. Soc. Am. 71, 744 (1981).

[53] C. C. Katsidis and D. I. Siapkas, General transfer-matrix method for optical multilayer systems with coherent, partially coherent, and incoherent interference, Appl. Opt. 41, 3978 (2002).

[54] C. L. Garrido Alzar, M. A. G. Martinez, and P. Nussenzveig, Classical analog of electromagnetically induced transparency, Am. J. Phys. 70, 37 (2002).

[55] Y. S. Joe, A. M. Satanin, and C. S. Kim, Classical analogy of Fano resonances, Phys. Scr. 74, 259 (2006).

[56] B. Gallinet, in Model of Coupled Oscillators for Fano Resonances, in Fano Resonances in Optics and Microwaves, edited by E. Kamenetskii, A. Sadreev, A. Miroshnichenko (Springer Book, New York, 2018), pp. 109-136.

[57] A. Lovera, B. Gallinet, P. Nordlander, and O. J. F. Martin, Mechanisms of Fano resonances in coupled plasmonic systems, ACS Nano 7, 4527 (2013). 